\title{
Adverse Selection and Liquidity Distortion in Decentralized Markets*
}

\author{
Briana Chang ${ }^{\dagger}$ \\ First Draft: Nov 1, 2010 \\ This version: May 31, 2011
}

\begin{abstract}
This paper studies the competitive equilibrium outcome in decentralized asset markets when both search friction and adverse selection play roles. In a dynamic environment with heterogeneous sellers and buyers, I show how adverse selection leads to the downward distortion of equilibrium market liquidity. The model predicts a strong link between the market liquidity and the underlying uncertainty stemming from adverse selection and therefore provides an explanation for the existence of massive illiquidity. As our setup captures two important dimensions in the trading market, price and liquidity, it shows how price and liquidity are jointly determined as an equilibrium outcome and further sheds lights on market segmentation. The framework also allows for a richer analysis of how sorting pattern is determined in such an environment and how different market segmentation may arise when sellers' motives for sale are unknown to the market.

Key words: Liquidity; Search frictions; Adverse selection; Over-the-Counter; Market segmentation
\end{abstract}

*I am indebted to Dale Mortensen for continuous support and encouragement. The paper also benefits from the discussion with Andrea Eisfeldt, Veronica Guerrieri, Arvind Krishnamurthy, Philpp Kircher, Alessandro Pavan, Robert Shimer, Martin Schneider, Randall Wright, Mirko Wiederholt, Simone Galperiti, and Martin Szydlowski.

†Northwestern University, Dept. of Economics, Email: bri.c@northwestern.edu 


\section{Introduction}

It is commonly believed that massive illiquidity has been a catalyst for the current financial crisis. Illiquid markets make it difficult for companies to access capital and likewise for investors to find a place to put their money to work. The real question is why markets remain illiquid even when there is a positive gain from trade. Furthermore, market liquidity usually presumes that there are buyers on the other side. However, as the recent crisis has demonstrated, the possibility of a "buyers strike" arises. This concept seems contradictory to the standard notion that prices should adjust downward to the level at which buyers will be willing to enter the market. A theory for these phenomena is developed. This paper studies a dynamic environment in which the decentralized trading markets are subject to both search frictions and adverse selection. Liquidity is endogenously determined in our model. With the existence of adverse selection, the model predicts that the equilibrium market liquidity will be downward distorted when compared to an environment with complete information. In fact, as an equilibrium outcome, prices will not adjust downward and fewer buyers will enter.

As lots of assets are traded in decentralized markets, traders must search for the counterparty in this environment. Hence, traders care about both the selling price as well as liquidity, which, in line with the Over-the-Counter literature, is defined as the expected search time. This definition here emphasizes the idea that market liquidity is provided by buyers on the other sides. How fast a seller can cash his assets will depend crucially on how many buyers are out there, ie, how tight the market is. Meanwhile, information asymmetries about the true value of an asset further results in strategic considerations when purchase and sales decisions are made. For example, the difficulty in assessing the fundamental value of asset-backed securities, which therefore leads to the adverse selection problem, has been one of the prevailing explanations for the recent crisis. The environment we consider here is relevant for any asset traded in decentralized markets and current owners tend to have private information about its quality, such as, current residents of the house, the banks who design the mortgage-backed securities, or the firms who own corporate assets, etc. It is important to emphasize that our setup captures two important dimensions in the trading market: price and liquidity. This distinguishes us from the long literature on adverse selection, focusing on price distortion which is simple one special kind of market distortion. As our setup allows traders to direct their search toward their preferred markets, market segmentation arises as an equilibrium outcome and, more importantly, equilibrium price and liquidity are jointly determined in each 
submarket. Although the impact of market illiquidity and the price discount is well understood, most analyses are conducted assuming their existence; little is known about why each occurs in the first place. The contribution is to characterize how price and liquidity are endogenously determined in decentralized markets and therefore sheds light on the effect of adverse selection on both the price and liquidity.

Our setup follows the competitive search equilibrium, where uninformed principals (buyers) post prices to attract informed agents (sellers) and agents direct their search toward their preferred market. It is well known that, given complete information, the outcome of a competitive search equilibrium put forth by Moen (1997) necessarily solves the social planner's problem. Moreover, Mortensen and Wright (2002) point out that one can think of the competitive search equilibrium outcome as if there is a third party (market maker) costlessly setting up the price and market tightness for each market. Extending this line of thought to the environment with adverse selection, this paper proposes that the equilibrium can be solved directly as a mechanism design problem, subject to both sellers' and buyers' optimality constraints. The approach is novel. While the current literature is only able to address a specific case, with this new approach, we can easily expand our analysis to a more general environment.

The first result shown in our basic model illustrates that the market with a higher quality asset will suffer a distorted market tightness when compared to the benchmark with complete information. The key intuition is that holding different quality assets results in different liquidity preference. This is essentially the mechanism behind this paper, which demonstrates an agent's type is revealed by his choice of market. The same mechanism also plays a role in a contemporaneous work by Guerrieri et al. (2010), who studies the competitive equilibrium outcome in a dynamic asset market without search frictions. In both Guerrieri et al. (2010) and our basic model, liquidity distortion works as a screening mechanism at the equilibrium. This outcome is unique and no pooling equilibrium, which involves the price distortion existing in the standard lemon model, can be sustained. Furthermore, in such an environment, I establish a strong link between the market liquidity (tightness) and the underlying uncertainty stemming from adverse selection. It is shown that the underlying dispersion, or more precisely, the possible range of underlying asset qualities, plays an important role in determining the equilibrium market liquidity: the higher the dispersion, the more illiquid the market. Contrary to the standard lemon model, what matters is the dispersion (range) of the asset instead of the expected value.

It is important to note, however, such an equilibrium outcome relies on the fact that 
buyers' willingness to pay aligns with sellers' preference over liquidity, which in general is not necessarily the case. For example, in a setting when sellers' motive for sale are unknown to the market, the types who are willing to wait longer are not necessarily the more valuable assets. Therefore, the corresponding mechanism must adjust. In a more general setting, we first identify the key condition for the existence of our baseline result and then show how a combination of both price and liquidity distortion arise when this condition does not hold. Utilizing this result, I investigate a setting when sellers' liquidity position are their private information and show how this setup can be nested in our general model. We show that a fire sale equilibrium can arise endogenously. In such an equilibrium, certain types of sellers will choose to enter a submarket that is liquid but has a heavy price discount. The contribution of this has is therefore twofold: First, it establishes how price and market liquidity are jointly determined by adverse selection and by the market's perceived motives for selling. Second, it demonstrates how different type of market segmentations arise as an equilibrium outcome. Interestingly and probably counter-intutiviely, private information on the sellers' motives for selling can actually improve market liquidity and possibly welfare.

Furthermore, as an application, the paper also contributes to the literature regarding the reallocations of firms' corporate assets (used capital). As the massive ongoing microeconomics restructuring and factor reallocation is crucial to aggregate performance, there is clearly a strong link between how the economy is doing and how well factors markets are functioning. Since our paper provides a micro-foundation for the reallocation frictions in the decentralized markets, it allows for a richer analysis of how the market friction responds to varied economic shocks. Both TFP and cross-sectional distribution of active firms are endogenously determined, as the outcome of markets. The implied illiquidity prevents resources from reallocating efficiently, generating a drop in productivity and a slow recovery, and, therefore, can result in substantial aggregate effects. It further provides an explanation as to why, as documented in the literature, such as Eisfeldt and Rampini (2006), capital reallocation is procyclical while the cross-sectional dispersion of the productivity is countercyclical.

Section 2 introduces the basic model and characterizes the equilibrium outcome. Section 3 show how the basic model can be generalized, allowing the extensions for heterogenous buyers and resale. Furthermore, it shows how the equilibrium behaves differently when monotonicity the matching value is relaxed and show how our method is still useful in such an environment. Section 4 considers an extension when sellers' liquidity position is unobserved by the market. A interesting phenomena arises: a fire sale equilibrium co- 
exists will illiquid submarkets. Section 5 and 6 discuss model implications and address issues on sorting pattern, efficiency and etc.

\section{Related Literature:}

The key ingredient of our model is the endogenous market liquidity stemming from search frictions and adverse selection in the competitive decentralized trading markets. A series of papers by Duffie, Garleanu and Pedersen - Duffie et al. (2005) and Duffie et al. (2007) - are the first to introduce search in models of asset market equilibrium. They build a dynamic asset-pricing model which shows how the equilibrium properties - allocation, prices, and bid and ask prices - depend on investors' search abilities and bargaining powers. Building on their model, the emerging literature studies the effects of liquidity in search models of asset pricing. ${ }^{1}$ Compared to this line of literature, the contribution of this paper is to analyze adverse selection in OTC markets with a setting of competitive equilibrium. To do that, we borrow the basic framework of the competitive search equilibrium developed in the labor search literature, put forth by Moen (1997), and Mortensen and Wright (2002); furthermore, this paper extends the framework to the environment in which sellers have private information about their asset quality.

Theoretically, our work is closest to Guerrieri et al. (2009), who apply the notion of competitive search equilibrium to an environment with adverse selection in a static environment. Both of our equilibrium concept shares the similar feature with the refined equilibrium concept developed in Gale (1992) and Gale (1996). In the above works, uninformed principles post (exchange) contracts, and it is important that the contract satisfies the sorting condition so that agents are screened. This assumption however does not hold here, as the contract space is limited to the payment in our trading environment. The focus of our framework is therefore on the effect of adverse selection on the extensive margin governed by the search friction, i.e market liquidity in the trading market. We first identify the condition under which an unique separated equilibrium is obtained, which ties our result to Guerrieri et al. (2009). Furthermore, we further characterize the equilibrium outcome in a more generalized environment when the monotonicity in the matching value is relaxed. In such an environment, the equilibrium then behaves differently from Guerrieri et al. (2009) as well as a contemporaneous work by Guerrieri et al. (2010), in which they study asset trading in a tree-economy without search friction but rationing. At a theoretical level, our work complements both Guerrieri et al. (2009) and Guerrieri et al. (2010) in the following respects. First of all, this paper provides an alternative way to characterize the equilibrium by establishing the decentralized competitive equilibrium as

\footnotetext{
${ }^{1}$ For example, Weill (2008), Lagos and Rocheteau (2009)
} 
a mechanism design problem. With this approach, we show that the equilibrium market liquidity can be characterized simply by a differential equation. This method is important as it further facilitates the analysis for a more general environment. Second, our setup is dynamic and is designed to handle two-sided heterogeneity. Finally, we investigate the case in which sellers' motives for sale are unknown to the market, with the possibility that the monotonicity condition does not hold. We show how a semi-pooling equilibrium, which behaves like a fire sale, arises endogenously in such an environment. This makes us distinct from Guerrieri et al. (2010), who consider a fire sale equilibrium by imposing all trades must take place at a common price. In our framework, a semi-pooling equilibrium arises endogenously when sellers' liquidity position is unknown.

Allowing for heterogenous buyers, the model sheds light on the sorting pattern. The generalization with heterogeneous buyers takes into account the competition among buyers as well as the diversity, which is the hallmark of economic exchange. It therefore sheds light on the sorting pattern. In particular, it is well known that, supermodularity in matching value is not enough to guarantee positive assortative matching (PAM) in a search framework. For example, Eeckhout and Kircher (2010) studies the sorting of heterogeneous agents in a competitive search trading market with complete information and identifies the condition under which PAM obtains. We show that, however, with adverse selection, positive assortative matching is guaranteed by the supermodularity in matching value. It is also important to note that the liquidity distortion stemming from the adverse selection also changes the trading pattern. A downward distortion of market liquidity, that is, a low ratio of buyers over sellers in the market, makes it hard for a seller to find a buyer but, on the other hand, makes it easier for a buyer to find a seller, shortening a buyer's wait time and expected search cost. Given that high-type buyers like to secure trade with high probability and they are willing to pay for this, the environment effectively makes a high type buyer more competitive and more sellers end up trading with high-type buyers.

Our work is also related to the long literature of adverse selection based on the classic market for lemon Akerlof (1970), where a pooling equilibrium and a price discount is obtained. Compared to this line of literature, our setting introduces another important dimension of the market distortion: liquidity. In fact, in our basic model, a pooling equilibrium can not be sustained as long as buyers have freedom to post the price and sellers can direct their search to each market (price). This also distinguishes us from a recent work by Chiu and Koeppl (2010), who studies trading dynamics in OTC in a random search setting and a pool equilibrium is obtained in their environment. As shown 
in our basic model, a fully separated equilibrium is the unique outcome in our setting, implying several distinct features: 1) All markets, in the equilibrium, are priced and open if and only if there is a positive gain from trade for the worst possible asset; 2) Nevertheless, some high quality asset markets are rather illiquid (or even close to frozen) so it is hard for sellers to get rid of their assets; 3) More importantly, different dispersion of the asset quality will have a first order effect on market illiquidity. Contrary to the standard adverse selection problem, which predicts that the equilibrium outcome highly depends on the expected value of assets because of the feature of a pooling equilibrium, what matters in our framework is the dispersion of asset quality. Furthermore, in a more general environment, we show how both price discount and liquidity distortion can arise resulting from different types of market segmentation. A submarket involving pooling exists and its behavior then shares similar feature with the literature. The novelty is show how different market segmentations may arise as an equilibrium outcome and the market distortion can have impact on both price and liquidity.

The market structure we developed can be easily applied to all decentralized asset trading markets. One particular example discussed in the paper is the market for firms' corporate assets. According to the empirical literature on this, such as, Maksimovic and Phillips (2001) and Warusawitharana (2008), these transactions improve the allocative efficiency of capital in the economy. ${ }^{2}$ Gavazza (2010) investigates how trading frictions vary with the thickness of the asset market by examining patterns in commercial aircraft markets and shows that market thinness generates frictions that are a large impediment to the efficient reallocation of capital. Our framework then provides an explanation for the market thinness and further allows for a richer analysis of how this market friction respond to varied economic shocks. At the macroeconomic level, Eisfeldt and Rampini (2006) first shows that this capital reallocation is procyclical while the dispersion of firms' productivity is countercyclical. Recent research ${ }^{3}$ on the impact of dispersion and uncertainty also confirms this result. In order to explain these facts, some macro-models introduce the idiosyncratic productivity shocks and capital adjustment cost into the representative agent's optimization problem. Bloom (2009) shows that, with existence of capital adjustment costs, higher uncertainty (measured as a shock to the second moment) expands firms' inactive regions because it increases the real-option value of waiting. This concern

\footnotetext{
${ }^{2}$ Warusawitharana (2008) documented that purchases and sales of operating assets by firms generated $\$ 162$ billion for share holders over the past 20 years.

${ }^{3}$ For example, Bachmann and Bayer (2009) shows that cross-sectional standard deviation of frim-level innovations in the Solow residual, value added is robustly and significantly countercyclical; meanwhile, the cross-sectional standard deviation of firm-level investment is procyclical.
} 
then slows down the reallocations from low to high productivity firms. Instead of relying on an exogenous adjustment cost, we further provides the micro-foundation for this friction. In our model, firms, who receive a negative shock, do want to exit but have a hard time finding an investor who is willing to buy their capital in the equilibrium. This idea therefore provides the explanation as to why few firms exit in bad time, as documented in Lee and Mukoyama $(2008)^{4}$.

\section{$2 \quad$ A Basic Model}

There is a continuum of sellers who own one asset with different quality indexed by $s \in S$, which is private information. Let $G(s)$ denote the measure of sellers with types weakly below $s \in S$ and assume that $S=\left[s_{L}, s_{H}\right] \subset R_{+}$. While holding the asset $s$, the seller enjoys a flow payoff $s$ but must at the same time pay a holding cost as long as the asset remains unsold. One can think of this as a simple way to model a seller's need to "cash" the asset. As explained in Duffie et al. (2007), we could imagine this holding cost to be a shadow price for ownership due to, for example, (a) low liquidity, that is, a need for cash; (b) high financing cost; (c) adverse correlation of asset returns with endowments; or (d) a relatively low personal use for the asset, for example, for certain durable consumption goods such as homes.

There is a large continuum of homogenous buyers (that is, we assume that the measure of buyers is strictly larger than sellers), who enjoy the asset, $s$, with a flow payoff $s$. In order to buy the asset, the buyer needs to enter the market to search for the seller, incurring a search cost, $k>0$ for the duration of the search. All agents are infinitely lived and discount at the interest rate, $r$. Time is continuous. As standard, traders are subject to the random matching function. The standard assumptions on the matching function in each potential market are imposed, i.e, $m(B, \mu)$ has constant returns to scale and $m_{B}>0, m(\mu, 0)=0, m_{\mu}>0, m(0, B)=0$, where $B$ and $\mu$ denote the measure of the buyers and sellers in each market. Hence, the market tightness of each market depends on the ratio of buyers to sellers in that market, $\theta=\frac{B}{\mu}$. Therefore, sellers meet

\footnotetext{
${ }^{4}$ Regarding macroeconomic implications, the paper is broadly connected to the literature emphasizing the macroeconomics of restructuring. As a survey of this line of literature, Caballero (2007) is a useful reference, both for theoretical frameworks and empirical evidence. The effect of financial friction or investment adjustment cost has been widely studied in the standard macroeconomic DSGE model. Compared to this line of the literature, we look closely at the source of friction existing in the decentralized market and provide a micro-foundation in order to analyze how this friction responds to different economic environment.
} 
buyers with the arrival rate $\frac{m(\mu, B)}{\mu}=m(\theta)=m\left(1, \frac{B}{\mu}\right)$, which is increasing in $\theta$, and buyers

meet sellers with the meeting rate $\frac{m(\mu, B)}{B}=\frac{m(\theta)}{\theta}$, which is decreasing in $\theta$. Particularly, throughout this paper, we assume that the matching function takes Cobb-Douglas form, $m(\mu, B)=M \mu^{1-\rho} B^{\rho}$ but it can easily adapt to a different search technology.

\subsection{Benchmark: Complete information}

We first establish the benchmark with complete information, which is the canonical competitive search model put forth by Moen (1997). In our particular setup, buyers simply post a trading price and sellers direct their search toward their preferred market. All traders have rational expectations in the equilibrium market tightness associated with each market. Moreover, following the interpretation of Mortensen and Wright (2002), one can imagine the competitive search equilibrium as if there is a market maker who can costlessly set up a collection $\Theta$ of submarkets. Each market can be characterized by a pair $(\theta, p)$, which is known ex ante to participants. Given the posting price and the market tightness in each market, each trader then selects the most preferred submarket in which to participate (search). With the assumption that there is perfect competition among market makers, the market maker's problem is then to maximizes traders' utilities.

Sellers' and buyers' expected utilities who enter the market with the pair $(\theta, p)$ can be expressed as follows, respectively:

$$
\begin{gathered}
r U(s, \theta, p)=s-c+m(\theta))(p-U(\theta, s)) \\
r U_{b}(s, \theta, p)=-k+\frac{m(\theta)}{\theta}\left(\frac{s}{r}-p-U_{b}(\theta, s)\right)
\end{gathered}
$$

With the assumption that the measure of buyers are larger than that of sellers, free entry condition must hold for buyers. Hence, $U_{b}=0$. With perfect information, market maker's optimization problems for each market for asset $s$ is:

$$
\begin{array}{r}
\max _{p, \theta} U(s)=\max _{p, \theta} \frac{s-c+p m(\theta)}{r+m(\theta)} \\
\text { st }: \quad U_{b}(s)=\frac{m(\theta)\left(\frac{s}{r}-p\right)-\theta k}{r \theta+m(\theta)}=0
\end{array}
$$

One can easily see that $\theta_{F B}$ solves following FOC:

$$
\frac{c}{k}=\frac{1}{\rho}\left(r \theta_{F B}^{1-\rho}+(1-\rho) \theta_{F B}\right)
$$


Notice that $\theta_{F B}$ is an increasing function of the cost ratio, $\frac{c}{k}$. Namely, it is relatively easier for sellers to meet the buyer, and it takes longer for the buyer to find the seller when the holding cost is higher. Also, the first best solution is independent of the asset quality. The intuition is clear since the gain from trade is simply the holding cost, which is independent of the asset quality. The price of each asset is then: $p^{F B}(s)=\frac{s}{r}-\frac{k \theta_{F B}}{m\left(\theta_{F B}\right)}$, the expected value of the asset minus the expected searching cost paid by buyers. One can easily check that IR constraint holds for all types of sellers. Obviously, first-best allocations can not be implemented in the environment with adverse selection. Facing the same market tightness, sellers always want to pretend a higher type so that they can get a higher payment.

\subsection{Equilibrium with Adverse Selection}

As in the complete information environment, buyers/sellers choose the price they would like to offer/accept, and we can imagine a separate market for each price. In equilibrium, one can think of there being a separate market associated with each price. Buyers and sellers congregate in the preferred market to search for the counterparty. For any posting price $p, \mu(p, s)$ is the measure of type-s who want to accept $p$, and $B(p)$ is the measure of buyers who want to offer $p$. Therefore $(\mu, B)$ describes the trading decisions of sellers and buyers.

Our equilibrium concept adopts the concept of refined Walrasian general-equilibrium approach developed in Gale (1992). Same spirit has been used in the competitive search equilibrium literature, such as Guerrieri et al. (2009)-single buyer's offer does not affect the sellers' overall expected utility and agents have rational expectations about the market tightness and the distribution of agents' types associated with each contractand Eeckhout and Kircher (2010), where the payoff of each individual is determined only by his own decision and by the distribution of trading decisions $(\mu, B)$ in the economy. Clearly, no trade takes place at prices below zero and above $J(\bar{s})$, and we define the set of feasible prices as $P=[0, J(\bar{s})]$. To define agents' expected payoff, let the function $\theta(\cdot): P \rightarrow[0, \infty]$ denote the expected market tightness. For the active markets, that is, $P^{*}=\{p \in P \mid \mu(p, s)>0 \cap B(p)>0\}$, the market tightness is therefore well defined along with buyers' and sellers' expected payoff, which can be expressed as following:

$$
\theta(p)=\frac{B(p)}{\int \mu(p, s) d s}
$$




$$
\begin{aligned}
r U_{b}(p, \mu, \theta(p)) & =-k+\frac{m(\theta(p))}{\theta(p)}\left(\int \frac{\tilde{s}}{r} \mu(\tilde{s} \mid p) d \tilde{s}-p-U_{b}\right) \\
r U(p, \theta(p), s) & =s-c+m(\theta(p))(p-U)
\end{aligned}
$$

The equilibrium expected utilities of seller $s$ at equilibrium then must satisfy:

$$
V(s)=\max \left\{\frac{s-c}{r}, \max _{p^{\prime} \in P^{*}} U\left(p^{\prime}, \theta\left(p^{\prime}\right), s\right)\right\}
$$

We now need specify the belief out of the equilibrium path, following the spirit of refined equilibrium used in the literature. A buyer who contemplates a deviation and offers a price $p$ which has not been posted, $p \notin P^{*}$, has to form a belief about the market tightness and the types he will attract. First of all, a buyer expects a positive market tightness only if there is a type of seller who is willing to trade with him. Moreover, he expects to attract the type s who is most likely to come until it is no longer profitable for them to do so. Formally, define:

$$
\begin{aligned}
\theta(p, s) & \equiv \inf \{\tilde{\theta}>0: U(p, \tilde{\theta}, s) \geq V(s)\} \\
\theta(p) & \equiv \inf _{s \in S} \theta(p, s)
\end{aligned}
$$

By convention, $\theta(p, s)=\infty$ when $U(p, \tilde{\theta}, s) \geq V(s)$ has no solution. Intuitively, we can think of $\theta(p)$ as a lowest market tightness for which he can find such a seller type. Now let $T(p)$ denote the set of types which are most likely to choose p:

$$
T(p)=\arg \inf _{s \in S}\{\theta(p, s)\}
$$

Therefore, this suggests that, given $\theta(p), p$ is optimal for every type $s \in T_{p}$ but not optimal for $s \notin T_{p}$. Hence, the buyer's assessment about $\mu(p, s)$ for any posting price $p$ needs to satisfy the following restriction:

For any price $p \notin P^{*}$ and type $\mathrm{s}, \mu(p, s)=0$ if $s \notin T_{p}$

Definition 1 An equilibrium consists of traders' allocation $(B, \mu)$, a function of seller's expected utilities $V(s)$, a market tightness function in each market $p, \theta(\cdot): P \rightarrow[0, \infty]$ defined in (2) and (3), such that the following conditions hold:

E1 (optimality for sellers): let

$$
V(s)=\left\{\frac{s-c}{r}, \max _{p \in P^{*}} \frac{s-c+m(\theta(p))}{r+m(\theta(p))}\right\}
$$


and for any $(p, s) \in P^{*} \times S, \mu(p, s)>0$ implies

$$
U(p, \theta(p), s) \geq U\left(p^{\prime}, \theta\left(p^{\prime}\right), s\right) \forall p^{\prime} \in P^{*}
$$

E2 (optimality for buyers and free-entry): for any $p \in P^{*}$

$$
0=U_{b}(p, \mu, \theta(p))
$$

; and there does not exist any $p^{\prime} \in P$ such that $U_{b}\left(p^{\prime}, \theta\left(p^{\prime}\right), \mu\right)>0$, where $\theta\left(p^{\prime}\right)$ and $\mu\left(p^{\prime}, s\right)$ satisfies (3) and (4).

\subsection{Characterization}

Following the interpretation of the market marker in our benchmark, we show that the equilibrium outcome can be characterized as the solution of a mechanism design problem which takes into account both sellers' and buyers' optimality condition. Intuitively, one can think of the market maker as the mechanism-deisgner who promises the price and the market tightness in each market so that sellers truthfully report their type, that is, condition $E 1$ has to hold. Moreover, a feasible mechanism must satisfy the market clear condition. In other words, the market tightness must equal the ratio of the measure of buyers who are willing to pay $\mathrm{p}$ to the measure of types-s sellers who are willing to accept p, which is characterized by (2). Meanwhile, given that buyers can post the price freely in the decentralized markets, any price schedule recommended by the market marker has to be optimal for buyers. Otherwise, buyers will deviate by posting price other than the ones recommended by the mechanism designer. This point is characterized by condition E2.

Overview of the solution: Our approach follows two steps: First, we characterize the set of feasible mechanism $\alpha \in A$, which satisfies $E 1$ and free-entry condition (Proposition 1). Second, we use $E 2$ to identify the necessary condition for which the solution to the mechanism can be decentralized in equilibrium (Lemma 1 and Lemma 2). This result enables us to pin down the unique candidate among the set of feasible mechanism $A$, which is a fully separating one. At the end, we show that this candidate is indeed the solution, that is, E2 and participating constraints are all satisfied.

To find out the set of mechanism that satisfies sellers' IC constraints, we setup the problem as a mechanism design problem (of a market maker). By the revelation principle, it will be without loss of generality to focus direct revelation mechanisms. A direct mechanism is a pair $(\theta, p)$ where $\theta: S \rightarrow R_{+}$is the market tightness function and a price 
function $p: S \rightarrow R_{+}$. The mechanism is interpreted as follows. A seller who reports his type $\hat{s} \in S$ will then enter the market with the pair $(\theta(\hat{s}), p(\hat{s}))$. Hence, the value of seller $s$ announces his type $\hat{s}$ can then be expressed as:

$$
r U(\hat{s}, s)=s-c+m(\theta(\hat{s}))(p(\hat{s}))-U(\hat{s}, s))
$$

For notation simplicity, denote the price $p(\theta)$ is the price of market with the market tightness $\theta$. The seller's optimal search problem can be rearranged as:

$$
V(s)=\left\{\frac{s-c}{r}, \max _{\hat{s}} U(\hat{s}, s)\right\}=\left\{\frac{s-c}{r}, \max _{\hat{s}} \frac{s-c+p(\hat{s}) m(\theta(\hat{s}))}{r+m(\theta(\hat{s}))}\right.
$$

where $U(\hat{s}, s)$ is the contingent values of agent $s$ entering the market $\theta(\hat{s})$. A seller can always choose not to participate and he will get his autarky utility, $\frac{s-c}{r}$ in that case. Notice that this is equivalent to set $m(\theta)=0$. For convenience, one can think of not entering the market as if choosing the market where $\theta=0$. The IC can be re-written as the requirement that $s \in \arg \max _{s^{\prime} \in S} U(\theta(\hat{s}), s)$. First of all, we can prove that any mechanism which satisfies $E 1$ can be characterized with following proposition:

Proposition 1 The pair of function $\{\theta(\cdot), p(\cdot)\}$ satisfies sellers' optimality condition (E1) if and only if following conditions are satisfied:

$$
\begin{gathered}
\frac{1}{r+m\left(\theta^{*}(s)\right)} \text { is non-decreasing } \\
V(s)=\frac{u(s)+p^{*}(s) \cdot m\left(\theta^{*}(s)\right)}{r+m\left(\theta^{*}(s)\right)}=V\left(s_{l}\right)+\int_{s_{l}}^{s} U_{s}\left(\theta^{*}(\tilde{s}), \tilde{s}\right) d \tilde{s} \\
V(s) \geq \frac{u(s)}{r}
\end{gathered}
$$

Proof. Standard proof in mechanism design literature. See Appendix. In this basic model, $u(s)=s-c$.

From free-entry conditions, $U_{b}(p, \theta, \mu)=0$, where $\mu(s, p)$ can then be found from $p(s)$, each active market $(p, \theta)$ then has to satisfy the following relation:

$$
p=\int \frac{s}{r} \mu(s \mid p) d s-\frac{k \theta}{m(\theta)}
$$

Proposition 1 and the free-entry condition then define the set of feasible mechanisms, A. Market clear condition is guaranteed by free-entry condition. Namely, buyers will 
entry until the "right" market tightness is satisfied. Moreover, let $V(\alpha, s)$ denote the expected payoff to a type-s seller under the mechanism $\alpha \equiv(p, \theta)$ Each mechanism $\alpha \in A$ is then composed of a price schedule $p^{\alpha}(\cdot)$,market tightness $\theta^{\alpha}(\cdot)$, and corresponding sellers' utilities $V^{\alpha}(\cdot)$.This set then includes all possible pooling as well as separating equilibrium. Nevertheless, not all of them can be sustained in the decentralized equilibrium. A decentralized equilibrium has to satisfy buyers' optimality condition. Hence, $(p, \theta, V(s ; \alpha))$ is only an equilibrium if there is no profitable deviation for buyers to open a new market $p^{\prime}$, where the off-equilibrium belief is specified in (3) and (4), as discussed earlier. When a buyer considers to open a new market $p^{\prime} \notin$ range of $P$, they expect to only attract the type who is most likely to come, $T\left(p^{\prime}\right)$, as defined (4). To facilitate the analysis, we first prove following lemma:

Lemma 1 Given any the mechanism $\alpha \in A$, which includes a price function $p^{a}: S \rightarrow R_{+}$, market tightness function $\theta^{\alpha}: S \rightarrow R_{+}$, and sellers utilities $V^{\alpha}: S \rightarrow R_{+}$, for any price $p^{\prime} \notin$ range of $p$, the unique type who will come to this market $p^{\prime}$ is given,

$$
\begin{aligned}
T\left(p^{\prime}\right) & =s^{+} \cup s^{-} \\
\text {where } s^{-} & =\inf \left\{s \in S \mid p^{\prime}<p^{\alpha}(s)\right\} \\
s^{+} & =\sup \left\{s \in S \mid p^{\prime}>p^{a}(s)\right\}
\end{aligned}
$$

Proof. Notice that $p^{\alpha}(\cdot)$ is non-decreasing for $\forall \alpha \in A .^{5}$ Therefore, $T\left(p^{\prime}\right)$ is uniquely defined $^{6}$. Namely, the type which is most likely to come is unique. For any $p^{\prime} \notin$ range $p^{\alpha}$, by definition, $\theta\left(p^{\prime}, s\right) \equiv \inf \{\tilde{\theta}>0: U(p, \tilde{\theta}, s) \geq V(s ; \alpha)\}$. Therefore, for any $p^{\prime}>V(s ; \alpha)$, $\theta\left(p^{\prime}, s\right)$ solves: $U\left(p^{\prime}, \theta, s\right)-V(s ; \alpha) \equiv G\left(p^{\prime}, \theta, s\right)$,

$$
\begin{aligned}
\frac{d \theta\left(p^{\prime}, s\right)}{d s} & =-\left(\frac{d G / d s}{d G / d \theta}\right) \propto \frac{1}{r+m\left(\theta^{\alpha}(s)\right)}-\frac{1}{r+m\left(\theta\left(p^{\prime}, s\right)\right)} \\
& =\left\{\begin{array}{l}
<0 \text { if } p^{\prime}>p^{\alpha}(s),\left(\because \theta\left(p^{\prime}, s\right)<\theta^{\alpha}(s)\right) \\
>0 \text { if } p^{\prime}<p^{\alpha}(s),\left(\because \theta\left(p^{\prime}, s\right)>\theta^{\alpha}(s)\right)
\end{array}\right\}
\end{aligned}
$$

\footnotetext{
${ }^{5}$ Suppose that $p(s)>p\left(s^{\prime}\right)$ for some $s<s^{\prime}$ in equilibrium. Given that $\theta(s) \geq \theta\left(s^{\prime}\right)(\because$ monotonic conditoin), obviously buyers is strictly better off going the market $p\left(s^{\prime}\right)$. Hence, $p(s)$ will not be opened in equilibirum. Contradiction.

${ }^{6}$ For the case when sellers are out of the market, that is, $\theta(s)=0$, for convenience, we define the corresponding $p(s)=\bar{P}+\varepsilon$ so that Lemma 1 is well defined in this case. Notice that, for any $\alpha \in A$, if $\theta(s)=0$, it cannot be case that $\theta\left(s^{\prime}\right)>0$ for $s^{\prime}>s$. Therefore, applying Lemma 1 in this case implies that for any price $p^{\prime}$ which is higher than the existing market price, $T\left(p^{\prime}\right)=s^{*}$, where $s^{*}$ is the marginal type who is indifferent to staying the market, where $V\left(s^{*}\right)=\frac{u(s)}{r}$.
} 
Recall that, posting a new price $p^{\prime}$, a buyer should expect the lowest market tightness $\theta\left(p^{\prime}\right)=\inf _{s}\left\{\theta\left(p^{\prime}, s\right)\right\}$ and the type $T\left(p^{\prime}\right)=\arg \inf \left\{\theta\left(p^{\prime}, s\right)\right\}$. Above relation then implies that, for example, if a buyer deviates to posting a new price $p^{\prime}$ which is lower than all the existing price, so that $s^{-}=s_{L}$ and $s^{+}=\varnothing$, he should attract only the lowest type, given that $\theta\left(p^{\prime}, s\right)$ is increasing in $\mathrm{s}$ and, therefore, $s_{L}=\arg \inf _{s} \theta(p, s)=T\left(p^{\prime}\right)$. Similar argument holds for any $p^{\prime} \notin$ range of $p^{\alpha}$.

With this condition, we can then prove following lemma:

Lemma 2 There is no pooling submarket in equilibrium.

Proof. See Appendix. Intuitively, a buyer can post a new price $p^{\prime}$ which is only slightly higher that the original pooling price. In that case, he only pays a little bit more but gets the best type in the original pooling for sure (as implied from lemma 1), which therefore generates a profitable deviation.

Lemma 2 then allows us to focus on a fully separating equilibrium. In each market, $(\theta, p, s)$, the price schedule then has to satisfy:

$$
p(s)=\frac{s}{r}-\frac{k \theta(s)}{m(\theta(s))}
$$

Substituting this payment schedule into $(I C F O C)$ :

$$
V(s)=\frac{s-c+\left(\frac{s}{r}-\frac{k \theta}{m(\theta)}\right) m\left(\theta^{*}(s)\right)}{r+m\left(\theta^{*}(s)\right)}=V\left(s_{l}\right)+\int_{s_{l}}^{s} U_{s}\left(\theta^{*}(\tilde{s}), \tilde{s}\right) d \tilde{s}
$$

One can then get differential equation of $\theta^{*}(s)$ :

$$
\left[c+\frac{k}{\rho}\left((\rho-1) \theta-\frac{r \theta}{m(\theta)}\right)\right] \frac{d \theta}{d s}=-\frac{\theta}{\rho r}(r+m(\theta))
$$

Therefore, the market tightness function $\theta^{*}(\cdot)$ has to the solution of $(6)$ subject to the monotonic condition, $(M)$ in order to satisfy the incentive compatible constraints and free-entry condition. Left hand side of (6) is monotonically decreasing in $\theta$ and reaches zero at $\theta^{F B}$. Therefore, for any initial condition $\theta_{0}>\theta_{F B}$, the solution will be explosive and violate the monotonic solution. (6) is a separable nonlinear first-order differential equation with a family solution form:

$$
s=C+\int \frac{1}{f(\theta)} d \theta
$$

where $f(\theta)=\frac{-\frac{\theta}{\rho r}(r+m(\theta))}{\left[c+\frac{k}{\rho}\left((\rho-1) \theta-\frac{r \theta}{m(\theta)}\right)\right]}$. One can understand the qualitative properties the solutions by constructing a simple phase diagram. The solution is illustrated as below, which can 
be understood by observing that for any $\theta \in\left(0, \theta^{F B}\right)$, a) $f(\theta)<0$; b) $f^{\prime}(\theta)>0$ and c) $\lim _{\theta \rightarrow 0} f(\theta)=0$.

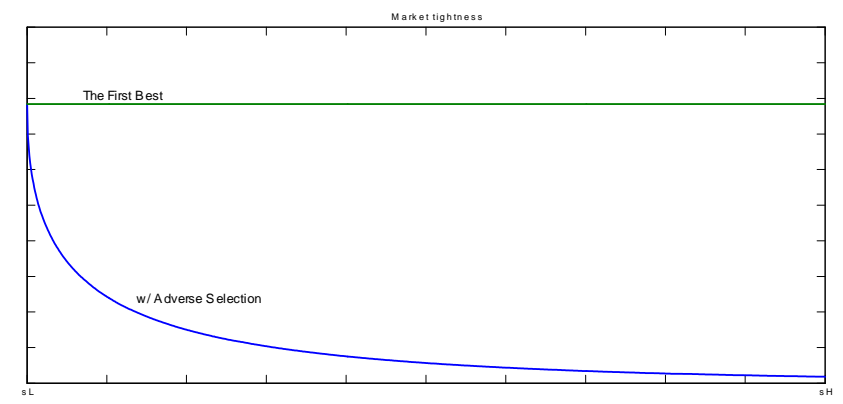

Equilibrium Market Liquidity $\theta^{*}(s)$

With the following initial condition ${ }^{7}$, we are able to pin down the unique solution which satisfies both sellers' and buyers' optimality constraints.

Lemma 3 The lowest type has to achieve his first-best utility. That is, the initial condition is:

$$
\theta\left(s_{L}\right)=\theta^{F B}\left(s_{L}\right)
$$

Proof. See Appendix for detail. The intuition is clear: a downward distorted market tightness is to preventing a lower-type from mimicking a higher-type. Therefore, it should be clear that there is no reason to distort $\theta$ for the lowest type.

The mechanism can be summarized as following. Because of the asymmetric information, sellers will then face a lower meeting rate, $\theta^{*}(s)<\theta^{F B}$ for all $s$ but get a higher transfer $p^{*}(s)=\frac{s}{r}-\frac{k \theta^{*}}{m\left(\theta^{*}\right)}>p^{F B}(s)$. There will be also less buyers participating the market, who needs to pay a higher price but with relatively high meeting rate. To note that, this result holds for any arbitrary distribution of sellers. Traders' participation and therefore, the trading volume, which is governed by the meeting rate, is endogenously determined. Also, we can easily check that IR constraint holds for all sellers ${ }^{8}$ and, indeed, buyers will

\footnotetext{
${ }^{7}$ One can see that standard condition of the uniqueness does not hold with this initial condition. In fact, there will be two solutions. However, the other soluction increases with s and therefore violates our monotonic condition.

${ }^{8}$ Define $G(s)=V(s)-\frac{s-c}{r}$ and $\left.\lim _{s \rightarrow \infty} G s\right)=0$, given $\lim _{s \rightarrow \infty} \theta(s)=0$. From $($ ICFOC $):$

$$
\frac{d g(s)}{d s}=\frac{1}{r+m\left(\theta^{*}(s)\right)}-\frac{1}{r}<0 \text { for all } \theta(s)>0
$$

Hence $V(s)>\frac{s-c}{r}$ for $\forall s<\infty$.
} 
not deviate by a opening market $p^{\prime}$ other than those which are already open. The argument is following: First, note that the price function is continuous. Denote $\left(p_{L}, p_{H}\right)$ as the lower bound and the upper bound support of function $p(s)$ constructed above. From Lemma 1 , if buyers post the price $p^{\prime}>p^{H}$, he will only attract the highest type. One can easily show that it involves more distortion and hence not profitable. Similarly, if posting

$p^{\prime}<p^{H}$, buyer will attract the lowest type and buyers' utilities will decreases due to the distortion. Namely, it confirms that no profitable deviation exists for buyers.

\section{Generalization}

The goal of this section is to study a more general economic environment where traders have different valuation of the asset. Similar as before, there is a mass of heterogenous sellers who are indexed by a type $s \in S$ that is sellers' private information. The flow payoff of the asset $s$ to the seller is now given by $u(s)$, where $u$ is a continuously differentiable function, $u: S \rightarrow R_{+}$The indices $s$ that are ordered such that they increase the utility of sellers: $u^{\prime}(s)>0$. On the other side of the market, there is a large mass of buyers. The flow payoff of an asset bought from seller $s$ is given by $h(s)$ and $h$ is a strictly positive function. I now make following assumptions on traders' preferences and will discuss how these assumptions can be relaxed in the later section.

Assumption 1: $h(s)$ is (1a) a continuously differentiable function and (1b) strictly increasing in $s, h_{s}(s)>0$

Assumption 2: $g(s)=h(s)-u(s)>0$ for $\forall s \in S$

Monotonicity in the matching value (1b) is an important assumption for our basic result. In particular, it is crucial to Lemma 2 under which we show that there is no pooling submarkets and an unique full-separated equilibrium is obtained under this assumption. As shown in our basic model, one can think of $s$ represent the quality of an asset, which gives both sellers and buyers a higher payoff. In the later section, I consider an environment when this assumption does not hold and show how the equilibrium outcome behaves differently. The second assumption simply guarantees that there is a gain from trade.

Given these two assumption, it is straightforward to see all our previous results holds, i.e. Proposition 1 and Lemma 1 to 3 . The only difference is now that equilibrium solution of the market tightness $\theta^{*}(s)$ needs to solve a more general form of differential equation, which is given by: 


$$
\left[\left(h(s)-u(s)-k\left(\frac{r+m(\theta)-\theta m^{\prime}(\theta)}{m^{\prime}(\theta)}\right)\right] \frac{d \theta}{d s}=-(r+m(\theta)) \cdot \frac{\theta}{\rho} \frac{h_{s}(s)}{r}\right.
$$

and the corresponding price schedule has to satisfies:

$$
p(s)=\frac{h(s)}{r}-\frac{k \theta^{*}(s)}{m\left(\theta^{*}(s)\right)}
$$

According to lemma 3 , the initial condition $\theta^{*}\left(s_{L}\right)=\theta^{F B}\left(s_{L}\right)$ and, in general, the first best market tightness solves:

$$
\frac{h(s)-u(s)}{k}=\frac{r+m(\theta)-\theta m^{\prime}(\theta)}{m^{\prime}(\theta)}
$$

Claim 1 The first best solution $\left\{\theta^{F B}(s), p^{F B}(s)\right\}$ is not implementable as long as $h_{s}>0$.

Claim 2 The solution $\theta^{*}(s)$ with the initial condition $\theta^{*}\left(s_{L}\right)=\theta^{F B}\left(s_{L}\right)$ is downward distorted compared to the first best, that is,

$$
\theta^{*}(s)<\theta^{F B}(s) \text { for } \forall s>s_{L}
$$

Proof. See Appendix.

Furthermore, observing from (8), one can see $\frac{d \theta}{d s}<0$, given that $\theta^{*}(s)<\theta^{F B}(s)$ and $h_{s}>0$.Therefore, $\theta^{*}(s)$ is decreasing and condition $(\mathrm{M})$ is satisfied. The equilibrium can be summarized as followings:

Proposition 2 Given $S=\left[s_{L}, s_{H}\right]$, a pair of $(p(s), \theta(s))$ that satisfies $(9),(8),(7)$ solves the market maker's constrained incentive-efficient problem. Moreover, this solution is the unique decentralized equilibrium where traders' allocations $\left(\mu_{t}, B_{t}\right)$ satisfy:

$$
\begin{aligned}
\mu_{t}\left(p\left(s^{\prime}\right), s\right) & =\left\{\begin{array}{c}
g_{t}(s) \text { if } s^{\prime}=s \\
0 \text { otherwise }
\end{array}\right\} \\
B_{t}(p) & =\left\{\begin{array}{c}
\mu_{t}(p, s) \cdot \theta(s) \text { if } \mu_{t}(p, s)>0 \\
0 \text { otherwise }
\end{array}\right\}
\end{aligned}
$$

where $g_{t}(s)$ is the measure of sellers' type s at $t$ 


\subsection{Heterogeneous Buyers}

The setup is now chosen to allow for heterogenous buyers in the market so that it can be easily applied to trading environments with two-sided heterogeneity. Many decentralized markets have this feature. Understanding the trading pattern is crucial since it determines the allocation and therefore welfare. For example, in the factor market, the resource allocation determines aggregate productivity. Different companies might have different technology to utilize the assets (machine or capital). Productivity of the assets is determined by assets allocation, which is mainly governed by both the pattern of trade and the equilibrium liquidity. With this generalization, the model further sheds light on the sorting pattern. We show that, supermodularity in the matching value is enough to guarantee positive sorting, which is a distinct feature compared to the environment without adverse selection.

Consider that there are two types of buyers, $b^{i} \in\left\{b^{h}, b^{l}\right\}$ and buyers' type are observable. For simplicity, we assume that the measure of each type is larger than the one of sellers and the outside option of buyer $b^{i}$ is given by $\phi\left(b^{i}\right)^{9}$. The flow payoff of an asset owned by buyer $b^{i}$ and bought from seller $s$ is given by $h\left(b^{j}, s\right)$, where $h$ shares the assumption as our basic model. The indices $s$ and $b^{i}$ that are ordered such that they increase the utility of sellers: $h\left(b^{h}, s\right)>h\left(b^{l}, s\right)$.For example, $h\left(b^{i}, s\right)$ represents the payoff produced by the firm with technology $b^{i}$ and asset quality $s$. The simple functional form widely used for a macroeconomic model with heterogenous firms is usually given by $h\left(b^{j}, s\right)=b^{j} s$, which can be seen as the productivity. Furthermore, we assume that there is complementarity in the matching function.

Assumption 3: $h_{s}\left(b^{h}, s\right)-h_{s}\left(b^{l}, s\right)>0$

Obviously, if $\phi\left(b^{h}\right)<\phi\left(b^{l}\right)$, sellers then always obtain higher value if trading with the higher type buyer and one can easily show that, facing resulting $(p, \theta)$, lower type buyer will not enter the market. In that case, the environment can be trivially solved just like as with homogenous buyers. The following analysis focus on the relevant environment in which both type of buyers are active in the market when there is no adverse selection. One can establish the benchmark similar as before. It is well known that the equilibrium outcome can be thought of as a competitive market maker who promises traders the price, the market tightness, as well as the trading pattern. The equilibrium will then consist of a price function $p^{F B}(s)$, a market tightness function $\theta^{F B}(s)$, trading pattern $j^{F B}(s)$ and

\footnotetext{
${ }^{9}$ This assumption is made to simplify the analysis. One can interpret this as a partial equilibrium where we take the level of buyers' utilites as given.
} 
the corresponding sellers' utility function $V^{F B}(s)$, which solve following the optimization problem:

$$
V^{F B}(s)=\max _{j, p, \theta}\left\{\frac{u(s)+m(\theta) p}{r+m(\theta)}: U_{b}\left(p, \mu, \theta, b^{j}\right)=\phi\left(b^{j}\right)\right\}
$$

The algebra detail is left in the appendix. In words, given buyer type $b^{i}$, one can solve the optimization problem as before. Similarly, the first best market tightness should be a function of the ratio of the gain from trade over the searching cost, which are type dependent and is denoted as $R(j, s) \equiv \frac{g(j, s)}{k+r \phi\left(b^{j}\right)}$. Let the function $V^{F B}\left(s, b^{j}\right)$ represent seller's utilities if traded with type $b^{j}$ under perfect information. The first best utilities $V^{F B}(s)$ can be seen as a upper envelope of $V^{F B}\left(s, b^{h}\right)$ and $V^{F B}\left(s, b^{l}\right)$. That is, $V^{F B}(s)=$ $\max _{j}\left\{V^{F B}(j, s)\right\}$. As it will become clear later, the interesting case is when there exists a marginal type $s^{F B} \in S$ who is indifferent to trading with high type and low type buyers and for a seller with assets $s<s^{F B}$, he will only trade with a lower type buyer and vice versa for sellers with assets $s>s^{F B 10}$. The following section therefore focuses on such an environment and discusses how other cases can be solved accordingly.

Apply our previous equilibrium definition but redefine $B$ as the measure of buyers $b^{j}$ entering the market $p$, where $B: P \times\{h, l\} \rightarrow R$. For the active markets, the market tightness is therefore well defined along with buyers' and sellers' expected payoff, which can be expressed as follows:

$$
\begin{gathered}
\theta(p)=\frac{\sum_{j} B\left(p, b^{j}\right)}{\int \mu(p, s) d s} \\
r U_{b}\left(p, \mu, \theta(p), b^{j}\right)=-k+\frac{m(\theta(p))}{\theta(p)}\left(\int \frac{h\left(b^{j}, \tilde{s}\right)}{r} \mu(\tilde{s} \mid p) d \tilde{s}-p-U_{b}\right) \\
r U(p, \theta(p), s)=u(s)+m(\theta(p))(p-U)
\end{gathered}
$$

Definition 2 An equilibrium consists of an allocation of traders $(\mu, B)$, a function of sellers expected utilities $V(s)$, and a market tightness function in each market $p, \theta(\cdot)$ : $P \rightarrow[0, \infty]$ defined in (10) and (3), such that the following conditions hold:

\footnotetext{
${ }^{10}$ It is clear from (??) that $R\left(h, s^{F B}\right)<R\left(l, s^{F B}\right)$, given $\phi^{h}>\phi^{l}$. Therefore, $\theta\left(h, s^{F B}\right)<\theta\left(l, s^{F B}\right)$ and $p\left(h, s^{F B}\right)<p\left(l, s^{F B}\right)$. Namely, there will be two separating markets for the asset $s^{F B}$. These two markets are different from the trading price and the liquidity, between which the seller $s^{F B}$ is indiscriminate. High type buyers will pay more for the good with shorter waiting time in one market and, vice versa for the low type buyers in the other market.
} 
E1 (optimality for sellers): let

$$
V(s)=\max _{p \in P^{*}} \frac{u(s)+p m(\theta(p))}{r+m(\theta(p))}
$$

and for any $(p, s) \in P^{*} \times S, \mu(p, s)>0$ implies

$$
U(p, \theta(p), s) \geq U\left(p^{\prime}, \theta\left(p^{\prime}\right), s\right) \forall p^{\prime} \in P^{*}
$$

E2 (optimality for buyers and free-entry): for any $p \in P$

$$
U_{b}\left(p, \mu, \theta(p), a^{j}\right) \leq \phi^{j}
$$

with equality if $p \in P^{*}$ and $B(p, j)>0$.

Clearly, IC constraints for sellers are the same as before, that is, Proposition 1 still holds. The only difference is that we need to make sure the buyers' optimality condition will hold for both types. In particular, facing the price and market tightness recommended by the market maker, a buyer will benefit neither from going to the markets which belong to the other buyers, nor from opening a market which has not been open. The mechanism can be interpreted as follows: given $(p(s), \theta(s))$, a seller reports his type $\hat{s}$ optimally; meanwhile, $j^{*}(s)$ denotes the sorting pattern recommended by the market maker, who recommends buyers $j^{*}(s)$ post the price $p(s)$, that is, entering the market $(p(s), \theta(s)$ ). The sets of types who trade with the lower type buyer, $\Omega_{L}=\left\{s: j^{*}(s)=l\right\}$, and of those who trade with the high type, $\Omega_{H}=\left\{s: j^{*}(s)=h\right\}$, are disjointed and satisfy $\Omega_{L} \cup \Omega_{H} \equiv S$. Then, define $s^{*}$ as the marginal type $j^{*}\left(s^{*}\right)=\{l, h\}$. Obviously, some lessons learned from the basic model are still applied: there is no submarket involving pooling under assumption 1b) and, hence, we can focus on the full separation on the sellers' sides. From buyers' view points, each market can therefore be characterized as a pair of $(p, \theta, s)$. Given $(p, \theta, s)$, buyers will choose to go to the preferred markets and expect to trade with seller $s$.

Moreover, once we identify the set of sellers who trade with buyers $j, \Omega_{j}$, the market tightness can be solved as in the case in which there is only one type of buyer $j$. Given $\Omega_{j}$, the solution of $\theta(s ; j)$ needs to the following differential equation, which is similar to (8) but taking into account that buyers' heterogeneity

$$
\left[\left(h\left(b^{j}, s\right)-u(s)-r \phi\left(b^{j}\right)\right)+\frac{k+r \phi\left(b^{j}\right)}{\rho}\left((\rho-1) \theta-\frac{r \theta}{m(\theta)}\right)\right] \frac{d \theta}{d s}=-(r+m(\theta)) \cdot \frac{\theta}{\rho} \frac{h_{s}\left(b^{j}, s\right)}{r}
$$


As before, the corresponding price schedule $p(j, s)$ is then pinned down with the free entry condition:

$$
p(s, j)=\frac{h\left(a^{j}, s\right)}{r}-\frac{\left(k+r \phi^{j}\right) \theta(s ; j)}{m(\theta(s ; j))}-\phi_{j}
$$

Notice that solutions can be easily characterized once we have the initial condition for $\theta(s ; j)$. Therefore, the key remaining task is essentially finding out the set $\Omega_{j}$, that is, the marginal type $s^{*}$ and identifying the initial condition $\left\{\theta_{L}^{0}, \theta_{H}^{0}\right\}$, which gives $\theta\left(s_{L} ; j\right)=\theta_{L}^{0}$ and $\theta\left(s^{*} ; j\right)=\theta_{H}^{0}$. For notation convenience, let $p^{j}(s), \theta^{j}(s)$ denote the price and the market tightness in the market with buyer type $j$. In equilibrium, it must be the case that the buyer $j$ will not enter the market where $j^{*}(s) \neq j$. Hence, following constraints must be satisfied:

$$
\begin{aligned}
U_{b}\left(p^{h}, \theta^{h}, s, b^{l}\right) & <\phi^{l} \text { for } j^{*}(s)=h \\
U_{b}\left(p^{l}, \theta^{l}, s, b^{h}\right) & <\phi^{h} \text { for } j^{*}(s)=l
\end{aligned}
$$

To facilitate the analysis, define $\tilde{\theta}(s)$ to solve the following:

$$
\begin{aligned}
\phi^{l} & =U_{b}\left(p^{h}, \theta, s, b^{l}\right) \\
& =U_{b}\left(p^{h}, \theta, s, b^{h}\right)-\frac{q(\theta)}{r+q(\theta)}\left(\frac{h\left(b^{h}, s\right)-h\left(b^{l}, s\right)}{r}\right) \\
& =\phi^{h}-\frac{q(\theta)}{r+q(\theta)}\left(\frac{h\left(b^{h}, s\right)-h\left(b^{l}, s\right)}{r}\right)
\end{aligned}
$$

where $q(\theta)=\frac{m(\theta)}{\theta}$. Given that $h\left(b^{h}, s\right)-h\left(b^{l}, s\right)$ increases with s, $\tilde{\theta}(s)$ increases with $s$. This function then plays an important role in determining buyers' incentive constraint. Entering the high-type buyers' markets, the difference in utilities gain is characterized by the second term, $\frac{q(\theta)}{r+q(\theta)}\left(\frac{h\left(b^{h}, s\right)-h\left(b^{l}, s\right)}{r}\right)$, which captures low types' disadvantage. The impact of this disadvantage is higher when the expected waiting time for buyers is shorter, that is, for the higher $q(\theta)$ and hence the lower $\theta$. As a result, for any $\theta<\tilde{\theta}(s)$, the low type will not mimic high type to enter the market. Similarly, when a high-type buyer contemplates entering a low-type market, he will only enter when $\theta<\tilde{\theta}(s)$ so that his advantage is high enough to compensate ${ }^{11}$. Hence, we can conclude the following claim:

Claim 3 In equilibrium, the market $(p, \theta, s)$ attracts high-type buyers but not low-type buyers if $\theta<\tilde{\theta}(s)$; similarly, the market $(p, \theta, s)$ attracts low-type buyers but not high-type buyers if $\theta>\tilde{\theta}(s)$.

\footnotetext{
${ }^{11}$ One can show that the utility of a high-type buyer entering a low-type market is: $U_{b}\left(p^{l}, \theta, s, a^{h}\right)=$ $\phi^{l}+\frac{q(\theta)}{r+q(\theta)} \frac{h\left(a^{h}, s\right)-h\left(a^{l}, s\right)}{r}$, which is bigger that $\phi^{h}$ iff $\theta<\tilde{\theta}(s)$.
} 
Denote the function $\theta_{j}^{F B}(s), V_{j}^{F B}(s)$ as the market tightness and sellers' utility, respectively, when trading with buyer $j$ with complete information. We next prove that the equilibrium can be characterized by following proposition.

Proposition 3 The unique solution to the mechanism is a market tightness function $\theta: S \rightarrow R_{+}$, a price schedule $P: S \rightarrow R_{+}$, a marginal type $s^{*}$, a pair of initial condition $\left\{\theta_{L}^{0}, \theta_{H}^{0}\right\}$, where:

$$
\begin{aligned}
\theta^{*}(s) & =\left\{\begin{array}{l}
\theta\left(s, l ; \theta_{L}^{0}\right), \text { for } s \leq s^{*} \\
\theta\left(s, h ; \theta_{H}^{0}\right), \text { for } s \geq s^{*}
\end{array}\right\} ; p^{*}(s)=\left\{\begin{array}{c}
p(s, l), \text { for } s \leq s^{*} \\
p(s, h), \text { for } s \geq s^{*}
\end{array}\right\} \\
V^{*}(s) & =V_{L}^{F B}\left(s_{L}\right)+\int_{s_{L}}^{s} \frac{u^{\prime}(\tilde{s})}{r+m\left(\theta^{*}(\tilde{s})\right)} d \tilde{s}
\end{aligned}
$$

where $\theta\left(s, j ; \theta_{j}^{0}\right)$ is the solution to (11) with the initial condition: $\theta\left(s_{L}, l\right)=\theta_{L}^{0}, \theta\left(s^{*}, h\right)=$ $\theta_{H}^{0}$, and corresponding $p(j, s)$ is defined in (12).

a) The initial condition $\theta_{l}^{0}$ :

$$
\theta_{l}^{0}=\theta_{l}^{F B}\left(s_{L}\right)
$$

b) The marginal types:

$$
s^{*}=\left\{\begin{array}{l}
s^{A}, \text { if } \tilde{\theta}\left(s^{A}\right) \geq \theta_{H}^{F B}(s) \\
s^{B}, \text { if } \tilde{\theta}\left(s^{A}\right)<\theta_{H}^{F B}(s)
\end{array}\right\}
$$

where $\left(s^{A}, s^{B}\right)$ is the unique ${ }^{12}$ solution to the following equation:

$$
\begin{aligned}
& s^{A}: \quad V(l, s)=V_{L}^{F B}\left(s_{L}\right)+\int_{s_{L}}^{s} \frac{u^{\prime}(\tilde{s})}{r+m\left(\theta^{*}\left(\tilde{s}, l ; \theta_{L}^{0}\right)\right)} d \tilde{s}=V_{H}^{F B}(s) \\
& s^{B}: \quad \tilde{\theta}(s)=\theta\left(s, l ; \theta_{L}^{0}\right)
\end{aligned}
$$

c) The initial conditions $\theta_{H}^{0}$ :

$$
\theta_{H}^{0}=\left\{\begin{array}{ll}
\theta_{H}^{F B}\left(s^{*}\right), & \text { if } s^{*}=s^{A} \\
\tilde{\theta}\left(s^{*}\right), & \text { if } s^{*}=s^{B}
\end{array}\right\}
$$

Proof. See Appendix.

As explained earlier, once we can separate the buyers from different markets, we can apply the method for homogenous buyers separately. Therefore, the equilibrium solution

\footnotetext{
${ }^{12}$ Observe that $\left(s^{A}, s^{B}\right)$ is unique (and all smaller than $s^{F B}$ ). Notice that, $V(l, s), V_{H}^{F B}(s), \tilde{\theta}(s), \theta_{H}^{F B}(s)$ are all well defined and monotonically increases in $s$ and $\theta\left(s, l ; \theta_{L}^{0}\right)$ is strictly decreasing in $s$.Given that $\tilde{\theta}\left(s_{L}\right) \leq \theta_{L}^{F B}\left(s_{L}\right)$ under the assumption $V_{L}^{F B}\left(s_{L}\right)>V_{H}^{F B}\left(s_{L}.\right) \Longrightarrow s^{B}$ always exists and is unique.
} 
is expected to be a combination of two. However, it has to be combined in a particular way so that traders' optimality conditions hold. In appendix, we prove that the constructed solution above is the unique solution. As expected, this solution is also the unique outcome of decentralized equilibrium and can be decentralized as follows:

Proposition 4 The solution to the mechanism $\left(\theta(s), p(s), s^{*}\right)$ is the outcome of decentralized equilibrium, where traders' allocations $\mu_{t}(s, p)$ and $B(p, j)$, sorting function $J^{*}(s)$ satisfies:

$$
\begin{aligned}
\mu_{t}\left(p\left(s^{\prime}\right), s\right) & =\left\{\begin{array}{c}
g_{t}(s) \text { if } s^{\prime}=s \\
0 \text { otherwise }
\end{array}\right\} \\
B_{t}(p, j) & =\left\{\begin{array}{c}
\mu_{t}(p, s) \cdot \theta(s) \text { if } \mu_{t}(p, s)>0 \text { and } J^{*}(s)=j \\
0 \text { otherwise }
\end{array}\right\} \\
J^{*}(s) & =h \text { for } \forall s>s^{*} ; J^{*}(s)=l \text { for } \forall s<s^{*}
\end{aligned}
$$

where $g_{t}(s)$ is the measure of sellers' type s at $t$.

\subsection{Extension: Resale}

When a firms thinks about buying the capital, he will need to take the business condition into account. If the environment is rather uncertain-for example, the financial source or the deploying technology is rather unstable-it will obviously decrease the firms' expected profit of the investment. When the firm receives a negative shock, he will then need to disinvestment, that's, he will then naturally become the seller in the market. Clearly, taking this situation into account, the firms' expected profit will also be a function of the resale value. To capture this idea, this section extends our main model to allow for the resale. To be precise, a firms' flow value of the capital decreases when hit by the negative shock and, in that case, he naturally becomes the seller in the market, which arrives at the Poisson arrival rate $\delta$.

$$
r J\left(b^{j}, s\right)=h\left(b^{j}, s\right)+\delta\left(V(s)-J\left(b^{j}, s\right)\right)
$$

where $V(s)$ is the expected value of the seller, as before. Obviously, the expression of a buyers' expected value searching in the market is the same as before and it has to equal to its outside option $\phi_{j}$ because of free-entry condition. All methods developed in our main model remain valid. As before, in order to solve for the equilibrium, we need 
derive following relations defined from the complete information case:

$$
\begin{aligned}
V^{F B}(s, j) & =\max _{\theta} \frac{r+\delta}{r}\left(\frac{u(s)}{r+\delta}+\frac{m(\theta)\left(\frac{h\left(a_{j}, s\right)-u(s)}{r+\delta}-\phi_{j}\right)-k \theta}{r+\delta+m(\theta)}\right) \\
\theta_{\delta}^{F B}(j, s) & =\arg \max V^{F B}(s, j)
\end{aligned}
$$

and the differential equation $\theta(s, j)$ and $p(s, j)$ derived from the sellers' IC constraints, given that he trades with the buyer $j$ :

$$
\begin{aligned}
& {\left[\left(h\left(a^{j}, s\right)-u(s)-(r+\delta) \phi^{j}\right)+\frac{k+r \phi^{j}}{\rho}\left((\rho-1) \theta-\frac{(r+\delta) \theta}{m(\theta)}\right)\right] \frac{d \theta}{d s} } \\
= & -\left(\frac{r+\delta+m(\theta)}{r+\delta}\right) \cdot \frac{\theta}{\rho}\left(h_{s}\left(a_{j}, s\right)+\frac{\delta u^{\prime}(s)}{r+m(\theta)}\right) \\
p(s, j)= & \frac{h(j, s)}{r+\delta}+\frac{u(s)}{r+\delta}+\frac{m(\theta)\left(\frac{h\left(a_{j}, s\right)-u(s)}{r+\delta}-\phi_{j}\right)-k \theta}{r+\delta+m(\theta)}-\frac{\left(k+r \phi_{j}\right) \theta}{m(\theta)}-\phi_{j}
\end{aligned}
$$

the modification to (13), $\tilde{\theta}(s)$ now solves:

$$
\phi_{h}-\phi_{l}=\frac{q(\theta)}{r+q(\theta)}\left(\frac{h\left(a_{h}, s\right)-h\left(a_{l}, s\right)}{r+\delta}\right)
$$

One can easily check that the previous version is simply the case when $\delta=0$. Obviously, the same approach can be applied. The only difference is that we now have a different differential equation and, different first best solution, different initial condition and different marginal type. Notice that the first best solution $\theta_{\delta}^{F B}(s)$ has to satisfy:

$$
(r+\delta) R(j, s)=\frac{h(j, s)-u(s)-(r+\delta) \phi^{j}}{k+r \phi_{j}}=\frac{1}{\rho}\left((r+\delta) \theta_{F B, \delta}^{1-\rho}+(1-\rho) \theta_{F B, \delta}\right)
$$

To note that $\theta_{F B, \delta}$ is decreasing in $\delta$. The intuition is clear since no buyers would want to enter the market if they need to sell it again soon. Since the trading surplus is decreasing in $\delta$, the higher $\delta$, the less entry and hence the lower equilibrium market tightness. Furthermore, let $g(s)$ denote the total measure asset supply $s$. Given the solution $\left(p^{*}(s), \theta^{*}(s)\right)$, the steady state can then be found. The measure of $H_{s s}(s)$ and $\mu_{s s}(s)$ at the steady state then equal $\frac{m\left(\theta_{s}\right)}{\delta+m\left(\theta_{s}\right)} g(s)$ and $\frac{\delta}{\delta+m\left(\theta_{s}\right)} g(s)$, respectively.

\subsection{Non-monotonicity in the Matching value}

The previous analysis shows that no pooling submarkets can exist in such an environment. This is a key feature of the model. It is important to note that it relies on the assumption, 
$h_{s}>0$. The intuition is that if a buyer strictly prefers a higher type $s$, he will benefit from posting a price which is slightly higher than the original pooling price so that no pooling can exist. Obviously, if this assumption is violated, a pooling equilibrium can then be sustained. Furthermore, as shown in the Proposition 1, any IC allocation $\theta^{*}(s)$ has to satisfy the condition (M). Observing from (11), if $h_{s}<0$, the solution $\theta^{*}(s)$ can then be increasing, which means that the allocation is no longer incentive compatible. Intuitively, the screening mechanism is a combination of a downward distorted liquidity and a upward price scheme. By the nature of the matching, the market marker needs to make sure buyers are willing to pay for the price, which relies on the fact that buyers also prefer a higher $s$. In other words, the screening mechanism works when buyers' willingness to pay aligns with sellers' willingness to wait. When these two values do not match, the screening is not implementable. In fact, as we will show in the next section, this setting has an interesting implication when sellers' motives for sale are their private information. The goal for now is to first establish how our previous analysis can be adjusted in a more general environment when the monotonicity condition is violated. Mainly, I show how a semi-pooling equilibrium can be constructed in a more general and abstract setting. In the later section, we apply this result to analyze the environment when sellers' motives for sale are their private information.

For simplicity, assume the case with homogenous buyers. The pooling outcome will obviously depend on the distribution and in general will not be unique. In fact, from our previous discussion, we know that any mechanism $\alpha=\left(p^{\alpha}, \theta^{\alpha}, V^{\alpha}\right)$ that promises a subset of sellers $S^{\prime}=\left[s_{1}, s_{2}\right] \subset S$ the same price schedule and market tightness (ie, semipooling) has to satisfy Proposition 1. Note that Proposition 1 remains intact regardless of the assumption on $h(\cdot)$. I now consider two approaches to establish a semi-pooling equilibrium, which mainly can be understood in the graphs below respectively. Notice that, depending on the distribution, in general the equilibrium is not unique and the condition for its existence is also identified below for each construction.

\subsubsection{Pooling Types}

The first approach can be constructed as follows: The first step is to "flatten" buyers'

valuations by pooling types. Intuitively, if we can flatten buyer's valuation over $s$ by bunching certain types together in a way that buyers' valuation is (weakly) monotonic in $s$, our previous analysis still applies (conditionally). However, flattening $h(\cdot)$ directly is convenient to work with but does not necessarily guarantee a non-increasing on $\theta^{*}(s)$. Therefore, the sufficient condition for which $\theta^{*}(s)$ is non-increasing is also identified as 
below.

Consider a continuously differentiable function $h(s)$, assuming that $h\left(s_{L}\right)<h\left(s_{H}\right)$ and the curve has a finite number of interior peaks on $\left[s_{L}, s_{H}\right]$. Pick a single interior peak $\hat{s}_{0}$, then there is also a single interior trough $\hat{s}_{1}$. As shown in the figure below, the inverse image of the interval $\left[h\left(\hat{s}_{1}\right), h\left(\hat{s}_{0}\right),\right]$ is composed of two intervals, $\left[s_{0}, \hat{s}_{0}\right]$ and $\left[\hat{s}_{1}, s_{1}\right]$, over which $h(\cdot)$ is increasing, and one interval, $\left[\hat{s}_{0}, \hat{s}_{1}\right]$ over which $h(\cdot)$ is decreasing. Let $\phi_{0}(h)$ and $\phi_{1}(h)$ denote the inverse functions of $h$ over the intervals $\left[s_{0}, \hat{s}_{0}\right]$ and $\left[\hat{s}_{1}, s_{1}\right]$. Last, let $\hat{h} \in\left[h\left(\hat{s}_{1}\right), h\left(\hat{s}_{0}\right)\right]$ solve:

$$
H(h) \equiv h-\int_{\phi_{0}(h)}^{\phi_{1}(h)} h(\tilde{s}) d G(\tilde{s})=0
$$

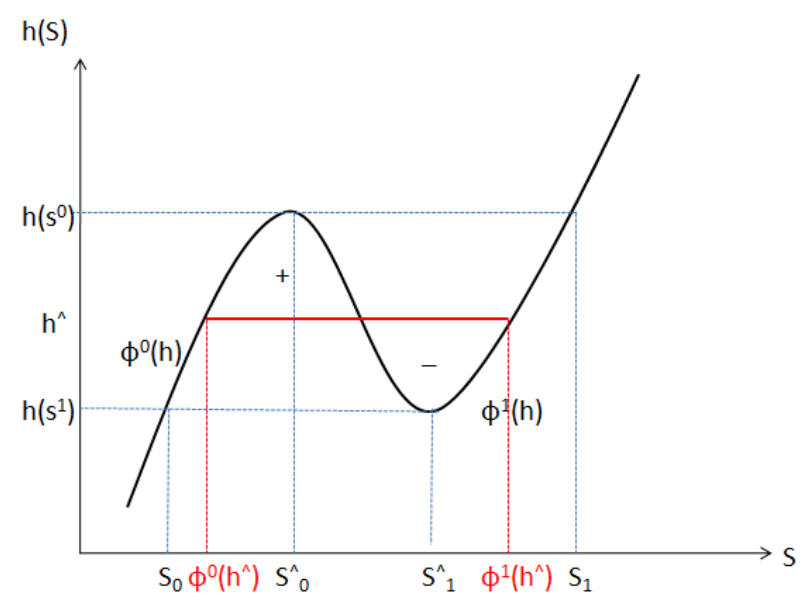

(A) Reconstructing $h(\tilde{s})$ by bunching types

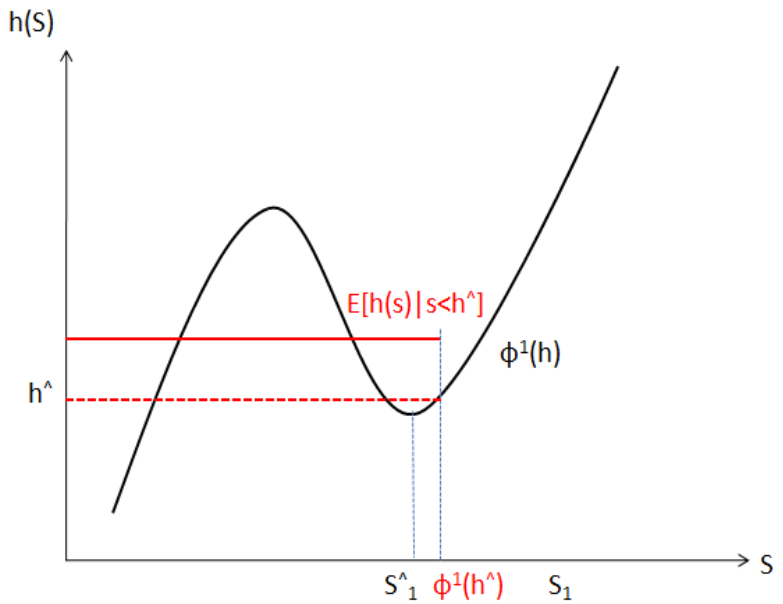

(B) Fire Sale Equilibrium

Given $\hat{h}^{13}$, a non-decreasing function $\tilde{h}(\cdot)$ can then be reconstructed as follows: let $\tilde{h}(s)=\hat{h}$ for $s \in\left[\phi_{0}(\hat{h}), \phi_{1}(\hat{h})\right]$ and $\tilde{h}(s)=h(s)$ otherwise. Suppose that there are more than one interior peak and we could independently different bunching levels where $\hat{h}_{1} \leq \hat{h}_{2}$, a non-decreasing function $\tilde{h}(\cdot)$ can be constructed in a similar way. If treating the two bunching regions separately yields $\hat{h}_{1}>\hat{h}_{2}$, we must then merge the two into a single bunching level. No matter what, if a non-decreasing function $\tilde{h}(\cdot)$ can be obtained: let $\theta^{*}(s)$ solves $(11)$ with the initial condition $\theta^{*}\left(s_{L}\right)=\theta^{F B}\left(s_{L}\right)$ at the interval $\tilde{h}(s)=h(s)$ for $\forall s \leq \phi_{0}(\hat{h})$ and, at the pooling interval, set $\theta^{*}(s)=\theta^{*}\left(\phi_{0}(\hat{h})\right)$ for $\forall s \in\left[\phi_{0}(\hat{h}), \phi_{1}(\hat{h})\right]$. Next, we should let $\theta^{*}(s)$ solves $(11)$ and set $\theta^{*}\left(\phi_{1}(\hat{h})\right)=\theta^{*}\left(\phi_{0}(\hat{h})\right)$ for the interval $\left[\phi_{1}(\hat{h}), s_{H}\right]$. However, the initial condition for $\theta\left(\phi_{1}(\hat{h})\right)$ has to satisfy the following condition so that

\footnotetext{
${ }^{13} \hat{h}$ might not be unqiue and its existence is garanteed as long as $h\left(s_{1}\right)>h\left(s_{L}\right)$
} 
the solution for the last interval is non-increasing:

$$
\theta^{*}\left(s_{0}\right)<\theta^{F B}\left(\phi_{1}(\hat{h})\right)
$$

Therefore, a semi-pooling equilibrium exists and is characterized by the constructed $\theta^{*}(s)$ and the corresponding price, $p^{*}(s)=\frac{\tilde{h}(s)}{r}-\frac{k \theta^{*}(s)}{m\left(\theta^{*}(s)\right)}$. With the above construction, one can easily see that free-entry condition is satisfied. In particular, in the pooling market, $E\left[h(s) \mid s \in\left[s_{0}, s_{1}\right]\right]=\hat{h}$ by construction. Although $\tilde{h}(\cdot)$ is not differentiable at the kink, ie. at the boundary point $s_{0}$ and $s_{1}$, the left and the right limit exists so (11) still applies. In other words, our method applies to any monotonically increasing $\tilde{h}(\cdot)$, even when $\tilde{h}(\cdot)$ has discontinuities of the first kind.

\subsubsection{Equilibrium with Fire Sales}

We now focus on a particular type of semi-pooling equilibrium, which has distinct features from the previous analysis. Consider a continuously differentiable function $h(s)$ on the interval $\left[s_{L}, s_{H}\right]$ and that is strictly increasing in $s$ after some point $\hat{s} \in\left[s_{L}, s_{H}\right]$ and let $\phi_{1}(h)$ denote the inverse function of $h$ mapping to $s \geq \hat{s}$, which is illustrated in the figure above.

Proposition 5 A semi-pooling equilibrium $\hat{h}$ with fire sales exists if following conditions hold:

$$
\begin{aligned}
\hat{\mu}(\hat{h}) & \equiv \int_{s_{L}}^{\phi_{1}(\hat{h})} h(\tilde{s}) d G(\tilde{s}) \geq \hat{h} \text { and } \\
V\left(s_{L}, \theta_{p}, p_{\mu}\right) & \geq V^{F B}\left(s_{L}\right)
\end{aligned}
$$

The marginal type is $s^{*}=\phi_{1}(\hat{h})$ and the pooling market with the market tightness $\theta_{p}$ is given by:

$$
\begin{aligned}
\theta_{p} & =\max _{\theta}\left\{\theta \mid V\left(s^{*}, p_{\mu}, \theta\right)=V^{F B}\left(s^{*}\right) \text { and } p_{\mu}=\frac{\hat{\mu}(\hat{h})}{r}-\frac{k \theta_{p}}{m\left(\theta_{p}\right)}\right\} \\
\theta^{*}(s) & =\left\{\begin{array}{cc}
\theta_{p} & \forall s \in\left[s_{L}, \phi_{1}(\hat{h})\right] \\
\theta\left(s ; \theta_{0}(\hat{s})\right) & \forall s \geq \phi_{1}(\hat{h})=s^{*}
\end{array}\right\}
\end{aligned}
$$

where, $\theta^{*}(s)$ denotes the solution of $(11)$ with the initial condition $\theta_{0}(\hat{s})=\theta^{F B}(\hat{s})$; and the equilibrium price follows:

$$
p(s)=\left\{\begin{array}{cc}
p_{\mu}=\frac{\hat{\mu}}{r}-\frac{k \theta_{p}}{m\left(\theta_{p}\right)} & \forall s \in\left[s_{L}, \phi_{1}(\hat{h})\right] \\
\frac{h(s)}{r}-\frac{k \theta^{*}(s)}{m\left(\theta^{*}(s)\right)} & \forall s \geq \phi_{1}(\hat{h})=s^{*}
\end{array}\right\}
$$


In words, there exists an marginal type $s^{*}=\phi_{1}(\hat{h})$ such that he is indifferent between trading in the pooling market and the market $\left(p^{F B}\left(s^{*}\right), \theta^{F B}\left(s^{*}\right)\right)$ by construction. In particular tightness $\theta_{p}$ is set to make sure this marginal type indifferent and furthermore, it is upward distorted, that is, $\theta_{p}>\theta^{F B}\left(s^{*}\right)$. For any sellers $s<s^{*}$, they enter the pooling market $\left(p_{\mu}, \theta_{p}\right)$; and for any $s>s^{*}$, they enter a separated market denoted by $(p(s), \theta(s))$ as in our basic model. The proof is straightforward from our previous discussion, so it is omitted. Notice that in such an equilibrium, there is a jump on the equilibrium price whenever $\hat{\mu}(\hat{h})>\hat{h}$. However, one can easily see that buyers will not deviate by posting any price $p \notin$ range $P$ as long as the stated conditions are satisfied. These two conditions guarantee that a buyer will not benefit from raising the price $\left(p^{\prime}=p_{\mu}+\varepsilon\right)$ to attract $\phi_{1}(\hat{h})=\hat{s}$ nor lowering the price $\left(p^{\prime}=p_{\mu}-\varepsilon\right)$ to attract $s_{L}$ as he obviously can not do better given $V\left(s_{L}, \theta_{p}, p_{\mu}\right) \geq V^{F B}\left(s_{L}\right)$ and $V(\hat{s})=V^{F B}(\hat{s})$.

\section{Obscure Motives for Sale}

In our baseline model, market liquidity essentially acts as a screening mechanism, similar to Guerrieri et al. (2009). As holding different quality assets results in different liquidity preferences, an agent's type is revealed by his choice of market. The crucial assumption in such an environment, however, is that agents' liquidity positions (i.e. the holding cost in our basic model) are observed. In an environment in which sellers' exact liquidity positions are not known by the market. For example, as pointed out by Tirole (2010), this situation is relevant when there are difficulties involved in apprehending banks' liquidity positions. Any incentive compatible mechanism must then accommodate this effect. Otherwise, sellers would benefit from appearing fragile in order to get a better price. In order to understand how market liquidity might be affected not only by adverse selection and by the market's perceived motives for selling, this section considers an extension when sellers' holding cost is not known to the market. In other words, there are two dimensions of sellers' types: the asset quality (common value) and the liquidity position (private value). The goal of this section is to understand how market liquidity and price are determined in such an environment. We first show how this setup can be nested in our general model and then discuss conditions that are crucial in order to determine whether the least distorted separation is the unique equilibrium outcome.

The setup is similar to our basic model but with the extension that a seller type now 
has two components: $z^{i}=\left(s^{i}, c^{i}\right) \in Z \equiv S \times C$. The support of $s^{i}$ is the real interval $S \equiv\left[s_{L}, s_{H}\right] \subset R_{+}$as before, but the support of $c^{i}$ is some arbitrary set $C$ which can assume discrete or continuous values. A seller's payoff of holding the asset is then governed by both the cash flow $s$ and his liquidity position $c$. Define type $\tilde{s}=s-c \in \tilde{S} \equiv\{s-c \mid$ $s \in S$ and $c \in C\}$, representing an agent's value of holding the asset. Clearly, the mechanism discriminates only on the basis of sellers' payoffs of owning the asset. In other words, two agents with the same type $\tilde{s}$ must enter the the same market, irrespective of any other unobservable characteristics that might differentiate the two agents in terms of their attractiveness to buyers. Therefore, one can understand this setup in our general model by following two reinterpretations. First of all, $\tilde{s}$ is now the relevant sellers' type. The utility of seller $\tilde{s}$, who reports his type $\hat{s}$, entering the market with $(\theta(\hat{s}), p(\hat{s}))$ can then be expressed as follows:

$$
r V(\hat{s}, \tilde{s})=\max _{\hat{s} \in \tilde{S}}\{\tilde{s}+m(\theta(\hat{s}))(p(\hat{s})-V(\hat{s}, \tilde{s})\}
$$

Second, as buyers care only about the asset quality (i.e the common value), a buyer's expected value of buying the asset from type $\tilde{s}$ can then be seen as $h(\tilde{s})=E[s \mid s-c=$ $\tilde{s}$, where $h: \tilde{S} \rightarrow R_{+}$. With the above interpretation, we can now apply our previous analysis. Obviously, what matters is $h(\tilde{s})$. Depending on the distribution, if $h(\tilde{s})$ is monotonically increasing, the equilibrium can be solved easily as before. In that case, sellers' private information on their liquidity positions essentially generates some noise, but the main result still hold. That is, a fully-separated equilibrium will be the unique equilibrium outcome. For a simple illustration, suppose $c^{i \sim} U\left(c_{L}, c_{H}\right)$ and $s^{i \sim} U\left(s_{L}, s_{H}\right)$, one can show that $h(\tilde{s})$ monotonically increases on the interval $\left[s_{L}-c_{H}, s_{H}-c_{L}\right]^{14}$. Hence, $\theta^{*}(\tilde{s})$ can be solved as before, and the corresponding price is given by $p(\tilde{s})=\frac{h(\tilde{s})}{r}-\frac{k \theta^{*}(\tilde{s})}{m(\theta(\tilde{s}))}$.

We now consider a more interesting case when $h(\tilde{s})$ is not monotonically increasing. Suppose there are two possible liquidity positions for sellers $C \equiv\left\{c_{H}, c_{L}\right\}$, where $c_{H}>$ $c_{L}>0$. Let $\lambda$ denote the probability of a seller who owns the asset $s$ suffers a worse liquidity position $c_{H}$. For simplicity, assume this probability is the same across asset quality $s^{i}$ and $s^{i \sim} U\left(s_{L}, s_{H}\right)$. The value of $h(\cdot)$ can then be understood in the left figure below:

\footnotetext{
${ }^{14}$ The function $h(\tilde{s})$ has two kinks at $\left(\tilde{s}_{1}, \tilde{s}_{2}\right)$, which, depending on the parameters, is given by: $\left\{\begin{array}{cc}\triangle c<\Delta s & \triangle c \geq \triangle s \\ \tilde{s}_{1}=s_{L}+\triangle c & \tilde{s}_{1}=s_{L}+\triangle s \\ \tilde{s}_{2}=s_{L}+\triangle s & \tilde{s}_{2}=s_{L}+\triangle c\end{array}\right\}$
} 


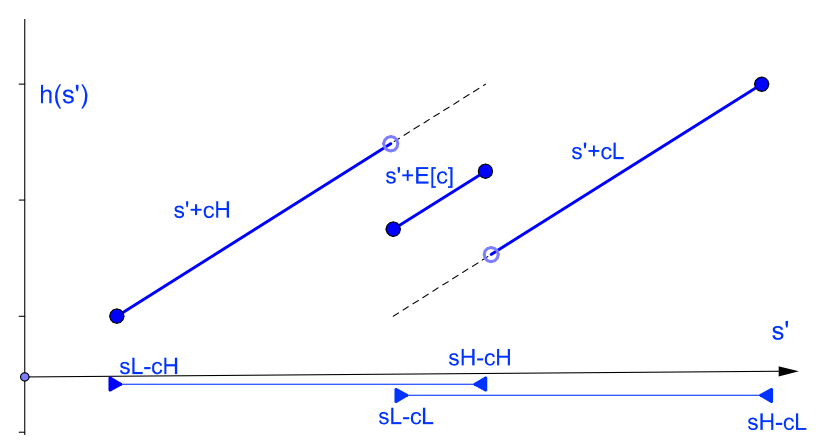

Buyers' value: $h(\tilde{s}): \tilde{S} \rightarrow R_{+}$

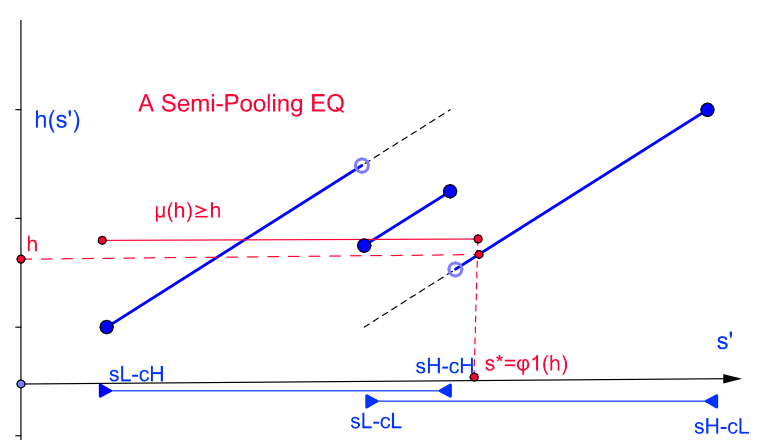

Constructing a Fire Sale EQ

As shown in the previous discussion, multiple semi-pooling equilibriums can exist. More importantly, a fire sale equilibrium can exist. Interestingly and probably counterintuitively, overall equilibrium market liquidity in fact can increase when sellers' holding cost are unobserved. Note that, however, an increase in market liquidity does not necessarily mean welfare improvement, which we will analyze in the later discussion. To illustrate this point, a fire sale equilibrium is now characterized as below and the condition for its existence is also established.

Buyers' value function $h(\tilde{s})$ is strictly increasing after $\hat{s}=s_{H}-c_{H}$. According to the Proposition 5, a fire sale equilibrium $\hat{h}$ can be found whenever there exists $\hat{h}$ such that the following conditions are satisfied: $\hat{\mu}(\hat{h}) \equiv \int_{s_{L}}^{\phi_{1}(\hat{h})} h(\tilde{s}) d \tilde{G}(\tilde{s}) \geq \hat{h}$ and $V\left(s_{L}, \theta_{p}, p_{\mu}\right) \geq$ $V^{F B}\left(s_{L}\right)$, which is illustrated in the right figure above. Notice that the set $\tilde{S}$ is the domain of both function $h(\tilde{s})$ and $\tilde{G}(\tilde{s})$. We start with the case when $s_{H}-s_{L}>c_{H}-c_{L}$ so that both $h(\tilde{s})$ and $\tilde{G}(\tilde{s})$ have full support, as it will become clear that a fire sale equilibrium always exists when $s_{H}-s_{L}<c_{H}-c_{L}$. Therefore the following characterization can be directly applied.

Proposition 6 Given $\lambda$, there exists $\bar{\triangle}(\lambda)>0$ and $\bar{k}>0$ such that, a fire sale equilibrium always exists when the dispersion of underlying liquidity position $\triangle c \equiv c_{H}-c_{L}>\bar{\triangle}(\lambda)$ and $k>\bar{k}$

For illustration purpose, an example of a fire sale equilibrium is characterized as follows: 

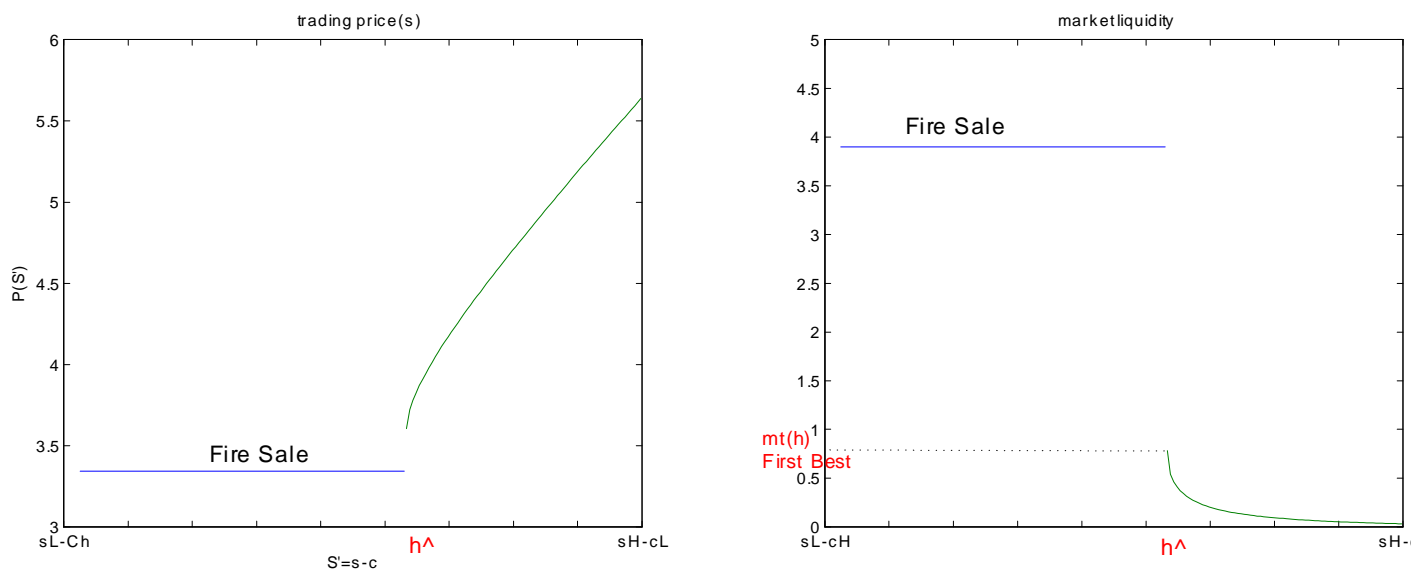

$$
s^{i \sim} U[2,3] ; c^{i} \in\{1,1.75\} ; \lambda=1 / 2
$$

We now give an example when assumed parameters satisfy the conditions, ie, $\exists \hat{h}>$ $s_{H}-c_{H}$, such that a fire sale equilibrium can be constructed as shown in the Proposition 5. The above figures shows the equilibrium price and market tightness $p: \tilde{S} \rightarrow R_{+}$and $\theta: \tilde{S} \rightarrow R_{+}$in each submarket. There exists a submarket which behaves like a fire sale, in which the price is heavily discount while market is liquid. This market attract all sellers $\tilde{s}<\phi_{1}(\hat{h})=s^{*}$. In this market, the price is given by:

$$
p^{p}=\frac{\mu(\hat{h})}{r}-\frac{k \theta^{p}}{m\left(\theta^{p}\right)}
$$

That is, buyers pay the average value among the pool of sellers, $\frac{\mu(\hat{h})}{r}$, minus the expected searching cost in this market. Hence, as in the standard pooling equilibrium, sellers with better assets sell their asset with a high price discount. Furthermore, there is another factor driving down the price- the upward distorted market tightness $\theta^{p}$. Sellers' utilities in such an equilibrium is shown below. Notice that in the pooling submarket, sellers with a worse asset are better off compared to benchmark as they effectively receive subsidies from sellers with a better asset. In the rest of submarkets, sellers with a better asset are worse off for the same reason in our basic model- downward distortion market liquidity. 


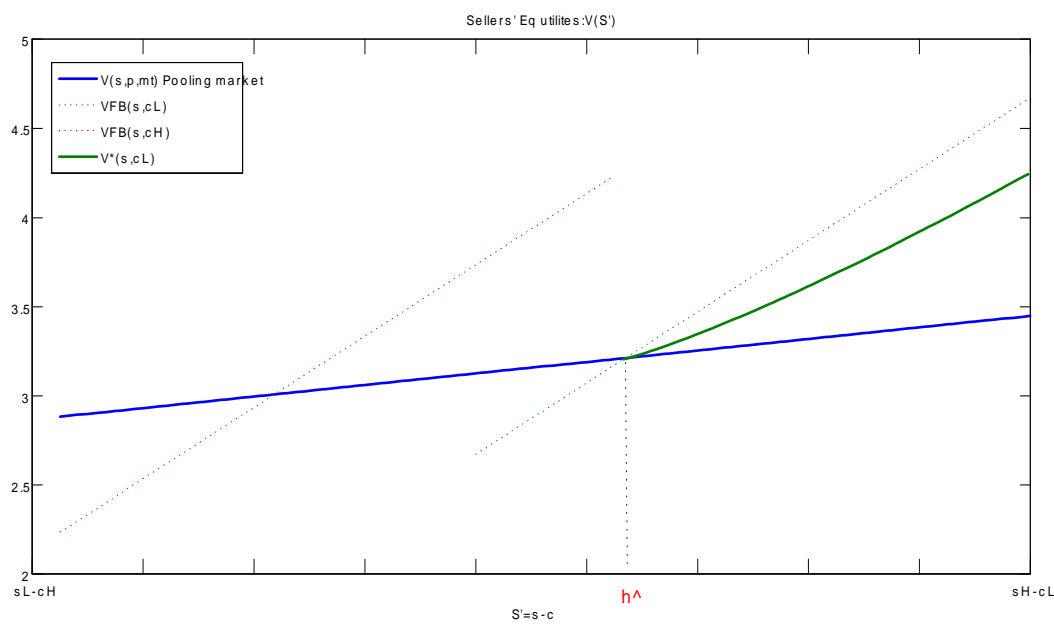

\section{Sellers' Utilites}

Comment: It is important to note that if in an environment where traders' liquidity positions are unobserved while resale is allowed, the original screening mechanism will also break down even though sellers are all homogenous. The reason is the same as above. Buyers are the ones who have a better liquidity position. In this case, a full pooling equilibrium can arise, which will then share similar features with the standard lemon problem. Taking into account the resale value, buyers utilities of owning asset $s$ can then be expressed as follows :

$$
J(s)=\max \left\{\max _{p \in P^{*}} \frac{s+m(\theta(p)) p}{r+m(\theta(p))}-T, \frac{s}{r}\right\}
$$

where $T$ represents the resale cost. In the equilibrium, there exists a marginal type such that $V\left(s^{*}\right)-T=\frac{s^{*}}{r}$. Buyers who bought asset $s \leq s^{*}$ will re-enter the market $(p(s+c), \theta(s+c))$; otherwise, they will keep their asset and enjoy its payoff $\frac{s}{r}$. The value of owning the asset $s$ can therefore be expressed as follows:

$$
J(s)=\left\{\begin{array}{cc}
V(s+c)-T & s \leq s^{*} \\
\frac{s}{r} & s>s^{*}
\end{array}\right\}
$$

One can understand our basic model as the case when the resale cost is high enough so that no buyers will re-enter and a unique equilibrium is obtained-a fully-separated one. On the other hand, when the resale cost is low (such that $\exists s^{*}>s_{H}-c_{H}$ ), a full pooling equilibrium exists and can be characterized by $(p, \theta)$ and the marginal type $s^{*}$. Conceptually, the equilibrium behaves similarly to the standard lemon problem so the 
detailed characterization is omitted here. The key message is that market segmentation in our framework is an endogenous outcome. Different market segmentation results in different forms of market distortion. It is important to note that, with adverse selection, different information setting on sellers' liquidity position (or their private value) will lead to different form of market distortion.

\section{Discussion}

\subsection{Remarks on Efficiency}

Does this decentralized equilibrium outcome necessarily solve the centralized planner's problem? The answer is obvious from our solution method. As explained earlier, among the set of feasible mechanisms $A$ defined from Proposition 1, the decentralized outcome is the one satisfying the buyers' optimality constraint, E2. Namely, given that buyers have the freedom to post new prices in the decentralized market, $E 2$ is the additional constraint compared to the social planner's problem. This logic clearly implies that a social planner can always (weakly) do better than the market. In fact, in our basic model, a full pooling equilibrium always achieves the first best welfare level as long as it is sustainable. The main reason is that the first best solution of market tightness is independent of types. A pooling equilibrium, simply subsidizing some at the expense of others, therefore does not incur any distortions as long as participating constraints of the highest types are satisfied. To be precise, a full pooling equilibrium maximizes social welfare as in the environment under complete information if and only if 1 ) the gain from trade is independent of types and 2) the IR constraint of the highest type is satisfied.

The above point then leads us to the next question: is the decentralized equilibrium outcome Pareto efficient? The answer can also be understood from our basic model. First of all, notice that the outcome of separating equilibrium does not depend on the distribution of types. On the other hand, traders' utilities in any kind of pooling equilibrium will obviously depend on the distribution. Intuitively, the highest type in a pooling equilibrium suffers a lower price because the market is only willing to pay the expected value of the asset. Nevertheless, in a separating equilibrium, he enjoys a much higher trading price but must suffer a long waiting time, decreasing his expected utilities. One extreme case would be that a full pooling equilibrium drives the highest type out of the market, which can happen when there are too many bad assets; therefore, the highest type is obviously better off in the separating equilibrium. In this particular case, the separating equilibrium 
is not Pareto ranked by the full pooling equilibrium ${ }^{15}$. Another extreme case would be that average quality is high enough so that even the highest type is better off in a full pooling equilibrium. The separating equilibrium then is Pareto ranked by the full pooling equilibrium. Hence, the answer to the efficiency properties of equilibrium will depend on the distribution assumed, which is actually a straightforward task once some particular distribution is given. This point then explains why the competitive search equilibrium is Pareto inefficient for some parameter values, as shown in Guerrieri et al. (2009). The important lesson is that the equilibrium outcome is not necessarily constrained Pareto efficient and the main reason is that pooling cannot be sustained even it is desirable due to the competitive nature of markets. And the distortions in market tightness resulting from separating equilibrium are rather costly.

\subsection{Remarks on Sorting Behavior}

Shi (2001) and Eeckhout and Kircher (2010) have shown that the complementarity in production is not enough to guarantee positive assortative matching (PAM) in an environment with complete information. The intuition is that, given that the social surplus increases with types, it could be optimal to match high-type seller with a low-type buyer by promising him a tight market, that is, a higher utilization. The above intuition still holds in our framework with complete information. However, with adverse selection, we prove that the supermodularity of the matching value necessarily induces PAM in the equilibrium.

Proposition 7 In the competitive search equilibrium with adverse selection, the equilibrium trading pattern $j^{*}(s)$ satisfies PAM, that is, for $s^{\prime}>s, j^{*}(s)=h \Longrightarrow j^{*}\left(s^{\prime}\right)=h$ under the assumption $h_{s}\left(a^{h}, s\right)-h_{s}\left(a^{l}, s\right)>0$,

Proof. Suppose Not. There exists $s^{\prime}>s$ such that $j^{*}(s)=h$ and $j^{*}(s)=l$. According to Claim 1, the equilibrium market tightness must satisfy: $\theta^{*}(s) \leq \tilde{\theta}(s)$ and $\theta^{*}\left(s^{\prime}\right) \geq \tilde{\theta}\left(s^{\prime}\right)$. Moreover, from the monotonic condition, $(M), \theta^{*}(s) \geq \theta^{*}\left(s^{\prime}\right)$. The above relation then implies $\tilde{\theta}(s) \geq \tilde{\theta}\left(s^{\prime}\right)$. This is a contradiction to the fact that $\tilde{\theta}(s)$ is strictly increasing with $s$ under the assumption $h_{s}\left(a^{h}, s\right)-h_{s}\left(a^{l}, s\right)>0$. (Recall $\tilde{\theta}(s)$ solves $\phi^{l}=\phi^{h}-$ $\left.\frac{q(\theta)}{r+q(\theta)}\left(\frac{h\left(a^{h}, s\right)-h\left(a^{l}, s\right)}{r}\right)\right)$

To understand this result, recall that the reason as to why a higher type can be better off when trading a low-type buyer is that he can be compensated by a higher utilization.

\footnotetext{
${ }^{15}$ However, depending on distribution, it could be ranked by a semi-pooling equlibrium.
} 
That is, given that a lower type buyer is more willing to wait, it could be optimal for a high type seller to choose to trade with a lower type buyer, enjoying a lower gain from trade but a tighter market compared to trading with a high type buyer. Hence, contingent on negative assortative matching (NAM), a high type seller must be compensated with a higher market tightness compared to low type sellers. This situation, however, can not be sustained in an environment with adverse selection, as it violates the monotonic condition. Namely, it is not incentive compatible for the sellers. Notice that in an environment with complete information, a high type seller prefer to trader faster as his gain from trade is higher. Nevertheless, with adverse selection, when all sellers are facing the same market price schedule and market tightness, a high type seller becomes the one who is more patient in the sense that he will prefer the combination of a higher price and a lower market tightness as contrary to a low type seller. This implies that it would be optimal to match a high type seller with a buyer who is more willing to offer a higher price and less willing to wait. Obviously, a high type buyer is more willing to do this. Hence, a lower type buyer no longer has his advantage to trade with a high type seller as in the case with complete information.

Our solution developed earlier starts with the environment with PAM and $V^{F B}\left(l, s_{L}\right)>$ $V^{F B}\left(h, s_{L}\right)$. However, according to the above Proposition, one should expect that those conditions can be relaxed. First of all, suppose $V^{F B}\left(h, s_{L}\right)>V^{F B}\left(l, s_{L}\right)$, so it is clear that $j^{*}\left(s_{L}\right)=h$ from Lemma 3 and, clearly, from the above Proposition, $j^{*}(s)=h$ for $\forall s \in S$. Hence, we can simply solve the model as if there are only high-type buyers in the market, regardless of positive or negative assortative matching under complete information. Suppose that we are now in the environment with NAM, that is, for $s^{\prime}>s, V^{F B}(l, s)-V^{F B}(h, s)>0 \Longrightarrow V^{F B}\left(l, s^{\prime}\right)-V^{F B}\left(h, s^{\prime}\right)>0$ and $V\left(l, s_{L}\right)>V\left(h, s_{L}\right)$, implying that only low type buyers are active in the case with complete information. Although we do not provide the formal solution for this case, our conjecture tells us that the solution should take similar pattern as the developed method. And, depending on the range of $S$, it could be the case that $j^{*}(s)=h$ for some $s^{\prime}>s$. The above argument shows that adverse selection essentially makes a higher type buyer more likely to stay the market compared to the case with complete information. Notice that this phenomenon can be understood for our main results as well, given that the marginal type decreases in the case of adverse selection, that is, $s^{*}<s^{F B}$. Hence, more sellers end up trading with high-type buyers with adverse selection.

This result might seem at first counter-intutive but, in fact, it is simply the flip side of market illiquidity. Adverse selection creates a downward distortion of market liquidity, 
that is, a low ratio of buyers over sellers in the market. This distortion makes it hard for a seller to find a buyer; on the other hand, it also makes it easier for a buyer to find a seller, shortening a buyer's wait time and expected search cost. Given that high-type buyers like to secure trade with high probability and they are willing to pay for this, the environment effectively makes a high type buyer more competitive, compared to low-type buyers.

\subsection{Search Frictions and Rationing}

One key feature of our results is that market liquidity acts as a screening mechanism in the setup of a competitive search equilibrium. Search friction has a natural interpretation in decentralized assets market and over-the-counter market, where traders' matching rate depends on how tight the market is. Interestingly, Guerrieri et al. (2010) independently obtained similar results in an environment without search frictions, where illiuqidity results from the rationing in each submarket. In the limiting behavior of their economy as the period length shortens, they show that the sale rate per unit of time can be seen as convergence to a Poisson arrival rate. Therefore, although with different interpretations, the trade-off faced by sellers in both our models are driven by different trading/matching rates and prices across markets. This then raises the question, what is the role of search friction and, economically, how and to what extent, is it different from rationing?

Notice that the approach of considering the limit economy as trading becomes more frequent does not shed light on the relation between search friction and rationing. In order to address this issue, one can understand the rationing as one special matching technology in the sense that the short side of the markets gets matched with certainty. This point has first made by Eeckhout and Kircher (2010), which considers a convergent sequence of search technologies in a static economy. Therefore, one way to show how search frictions vanish is to apply their approach in a discrete time version of our model and shows how the distortion varies as search friction vanishes. Here, we propose another way to see how search friction vanishes directly in our original continuos time setup. As show in Guerrieri et al. (2010), as an equilibrium outcome, buyers always match with probability one as they're the short side of the market. What is similar is that buyers in our model can trade relatively fast when facing a downward distorted market tightness. The key difference, however, lies on the fact that buyers in their model do not care about the market tightness conditional on they're at the short side of the market. Effectively, at the equilibrium, there is no congestion effect on the buyers' side. This effect is governed by 
the elasticity of the matching function with respect to measure of buyers in the standard codd-douglas matching function, that is, the parameter $\rho$ in our model. Therefore, one can understand the result in Guerrieri et al. (2010) as the limit economy in our model when $\rho \rightarrow 1$. Notice that the first best market tightness increases with $\rho$ as buyers care less about the market tightness. Hence the first best market tightness should increase. At the limit, the arrival rate for the lowest type goes to infinity when $\frac{c}{k}>1$, which corresponds to the standard discrete setup where the trading probability equals one (no rationing) given the gain from trade is larger than the entry cost.

\section{Implications}

\subsection{The Dispersion of Asset Quality}

As shown in the previous analysis, the equilibrium solution does not depend on the assumed distribution. In particular, given that the equilibrium market tightness is pinned down by the initial condition - which must equal the first best market tightness of the lowest quality asset - the dispersion, more precisely, the possible range of underlying asset quality plays an important role in determining the market liquidity. This implication can be understood from the basic model, even when the gain from trade is constant across assets. The impact of asset range on the market liquidity is summarized by the following proposition.

Proposition 8 Given any submarket with asset quality $s$, its resulting equilibrium liquidity, $\theta^{*}(s)$, increases with the quality of the worst asset of the whole market $s_{L}$. Formally, let $\theta\left(s ; \theta_{0}\left(s_{L}\right)\right)$ be the solution of (6) satisfying the initial condition $\theta\left(s_{L}\right)=\theta_{0}$. For any $s_{L}^{\prime}<s_{L}$, then

$$
\theta\left(s ; \theta\left(s_{L}^{\prime}\right)\right)<\theta\left(s ; \theta\left(s_{L}\right)\right) \text { for } \forall s
$$

Proof. Denote $\theta_{0}^{\prime}=\theta\left(s_{L}, \theta_{0}\left(s_{L}^{\prime}\right)\right)$, representing the equilibrium liquidity of market $s_{L}$ when the worst asset quality is $s_{L}^{\prime}$. Given that $\theta_{0}^{\prime}<\theta_{F B}\left(s_{L}\right)=\theta_{0}\left(s_{L}\right)$, the proposition is then simply a result of the Comparison Theorem.

It is important to note that this effect only exists in an environment with adverse selection, as we have shown that, with complete information, market liquidity is only a function of its own gain from trade. However, with adverse selection, the market liquidity of all submarkets are connected in the sense that the liquidity in each serves as a screening device to separate assets so that buyers can make sure the asset is worth the paying price. This result then links the impact of underlying asset distribution and market liquidity. 
Another interpretation of this result is the impact of transparency. To this end, we now allow assets to be subject to varying severities of the adverse selection problem. Formally, there are two different asset markets $i \in\{a, b\}$ and the payoff of each asset can be expressed as:

$$
d_{i}=y_{i}+\sigma_{i} s
$$

where $\left(y_{i}, \sigma_{i}\right)$ are publicly observed and $s$ is the owners' private information, where $s \in\left[s_{L}, s_{H}\right]$ with some distribution $G(s)$. Since $\left(y_{i}, \sigma_{i}\right)$ are publicly observed, we can imagine there are two separate markets for each asset, and buyers can choose to go to one of them. Each market can now be characterized as $\left(p^{i}, \theta^{i}\right)$, where $i \in\{a, b\}$.We can use similar methods to solve for $p^{i}\left(s ; \sigma^{i}\right)$ and $\theta^{i}\left(s ; \sigma^{i}\right)$. Clearly, a higher $\sigma_{i}$ has an similar effect to that resulting from a higher dispersion. It can be shown easily that the more transparent asset is more liquid. That is, for any $\sigma_{b}<\sigma_{a}, \theta^{*}\left(s ; \sigma_{b}\right)>\theta^{*}\left(s ; \sigma_{a}\right) \forall s$.

As the key element in our model is endogeny of market liquidity, the result also sheds light on the patterns in cross market liquidity. One important question which has been asked in the literature is, why assets paying similar cash flow can have significant differences in their liquidity. We can answer this question from the comparative static exercise above. Loosely speaking, an uncertainty of underlying asset quality has a significant effect on the overall market liquidity, even when the expected value remains unchanged. It therefore implies that assets paying identical cash flows can differ significantly from their liquidity, transaction cost, and price. All of above are endogenously determined in the equilibrium and can be understood as follows:

$$
p^{i}(s)=\underbrace{\frac{d_{i}}{r}}_{\begin{array}{c}
\text { present } \\
\text { value }
\end{array}}-\underbrace{\frac{k \theta^{i}\left(s ; \sigma^{i}\right)}{m\left(\theta^{i}\left(s ; \sigma^{i}\right)\right)}}_{\begin{array}{c}
\text { transaction } \\
\text { cost }
\end{array}}+\underbrace{\frac{\delta}{r}\left(\frac{c+k \theta^{i}\left(s ; \sigma^{i}\right)}{r+\delta+m\left(\theta^{i} ; \sigma^{i}\right)}\right)}_{\begin{array}{c}
\text { resale } \\
\text { value }
\end{array}}
$$

For any two asset paying the same cash flows, that is, the same $\frac{d}{r}$, their transaction costs as well as resale values, which are both functions of their own liquidity $\theta^{i}\left(s ; \sigma^{i}\right)$, are pinned down by each asset's own underlying dispersion. They can therefore result in a completely different liquidity pattern.

\subsection{Policy Implication: Buyback}

This section focuses on the buyback policy, as we know that cleaning up the toxic asset will show a significant improvement in overall market liquidity. The idea of cleaning up the toxic asset in the market is not new. In particular, Tirole (2011) shows that 
the intervention needs to take into account traders' participation constraints and the government always strictly overpays for the worst asset. In our framework, since traders can choose either to trade their assets in the over-the-counter market specified earlier or to join the government's scheme, traders only join the scheme if and only if they are leaving the market. Hence, though we do know that cleaning up the toxic asset would improve the market liquidity, what is important is to understand what price has to be paid in order to clean the market. Suppose now the government offers the price $p_{g}$ to whomever shows up in the discount windows. Anticipating that traders' utilities would increase in the future after government intervention, the original price and market tightness is then no longer incentive-compatible. Intuitively, a seller can now choose to hold on to the asset and claim a higher type in the future. Therefore, to solve for the equilibrium, a mechanism designer needs to take into account that sellers participate in the scheme only if they get at least as much as what they would have obtained in the decentralized market.

In the equilibrium, the sets of types who join in government intervention, $\Omega_{g}$, and of those who stay in the decentralized market, $\Omega_{d}$, are disjointed and satisfy $\Omega_{g} \cup \Omega_{d} \equiv S$. Agents' utilities can be expressed as:

$$
V(s)=\max \left\{\frac{s-c}{r}, \max _{p^{\prime}} U\left(p^{\prime}, \theta\left(p^{\prime}\right), s\right), p_{g}\right\}
$$

One can therefore pin down the condition for the marginal participant type $s^{*}$ :

$$
V\left(s^{*}\right)=p_{g}
$$

That is, the marginal type has to be indifferent between trading in the OTC market and obtaining the transfer from the government right away. Let $\left(p^{*}, \theta^{*}\right)$ denote the price and the market tightness that the marginal participant type will be facing if he goes to the market. Obviously,

$$
\begin{aligned}
& U_{g}(s)=p_{g}>\max _{p^{\prime}} U\left(p^{\prime}, \theta\left(p^{\prime}\right) ; \Omega_{d}\right) \text { for } \forall s<s^{*} \\
& U_{g}(s)=p_{g}<\max _{p^{\prime}} U\left(p^{\prime}, \theta\left(p^{\prime}\right) ; \Omega_{d}\right) \text { for } \forall s>s^{*}
\end{aligned}
$$

Also, from the previous discussion, we know how to solve the equilibrium outcome $p(\cdot), \theta(\cdot)$, given $\Omega_{d}$. The key task is to pin down the marginal type, which is characterized as follows:

Proposition 9 A competitive search equilibrium with government buyback price $p_{g}$, is a marginal participant type $s^{*}$ which solves

$$
V\left(s^{*}\right)=p_{g}=V^{F B}\left(s^{*}\right)
$$


and a pair of $\left(p\left(s ; \Omega_{d}\right), \theta\left(s ; \Omega_{d}\right)\right)$ that satisfies $(5),(6),(7)$, as a solution to the market maker's constrained incentive-efficient problem.

The figure below shows traders' utilities with the buyback policy $p_{g}$, represented by the red line. Given any price offered by the government, the marginal types s has to solve $V^{F B}\left(s^{*}\right)=p_{g}$, represented by the intersection of $p_{g}$ and the green line, which in turn is represented by $V^{F B}(s)$.

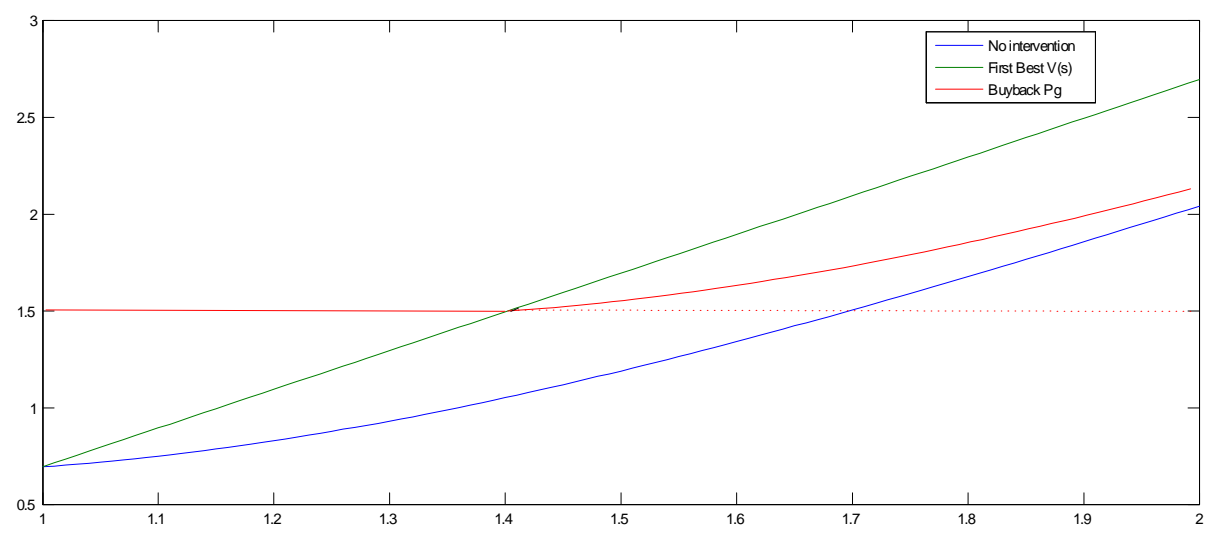

Equilibrium with Buyback Policy $P_{g}$

\subsection{Reallocation and Macroeconomic Performance}

As the massive ongoing microeconomics restructuring and factor reallocation is crucial to aggregate performance, there is clearly a strong link between how the economy is doing and how well factors markets are functioning. To this end, this section focuses on the reallocation of firms' corporate assets, given that capital is one of the important factors determining aggregate productivity. As documented in the empirical literature, changes in ownerships of firms' corporate assets - for example, product lines, plants, machines, and other business units - affect productivity. More precisely, capital typically flows from less to more productive firms, and the productivity of acquired capital increases. Furthermore, as suggested by the empirical findings, market thinness generates frictions that are a large impediment to the efficient reallocation of capital, even within a well-defined asset class, in which capital is moderately specialized. We then provide a possible explanation as to why the market for used capitals is relatively thin. Severe trading delays would result in resource mismatch and have a negative impact on aggregate performance. This paper then 
elucidates the underlying source of market frictions and its link to economic fluctuation

To illustrate our result, we now specify the function governing traders' payoff, nested in our general framework. The flow value of the capital is simply the product of the capital quality $s$ and its use of technology $a_{j}$, that is, $h\left(a_{j}, s\right)=a_{j} s$. More productive firms will be the natural buyers. In the economy, there are two profitable technologies $j \in\{H, L\}$,where $a_{H}>a_{L}$, and the owner of the technology $j$ has the outside option $\phi_{j}$, where $\phi_{H}>\phi_{L}$. Firms who receive a negative shock, at the arrival rate of $\delta$, become unproductive and only produce a flow payoff $a_{o} s$, where $a_{o}<a_{L}<a_{H}$, that is, $u(s)=a_{o} s$. Let $H_{t}^{j}(s)$ represent the measure of capital $s$ owned by the firm with technology $j$ and let $\mu_{t}(s)$ denote the measure of sellers who own capital $s$. The aggregate productivity of the capital can then be defined as follows:

$$
\bar{A}_{t}=\int\left\{a_{o} s \mu_{t}(s)+a_{l} s H_{t}^{l}(s)+a_{h} s H_{t}^{h}(s)\right\} d s
$$

Therefore, both the aggregate TFP and the cross-sectional distribution of active firms are endogenously determined in our model, crucially depending on how well the economy can allocate its resource to better hands. Interestingly, probably counter-intuitively, at the macro level, Eisfeldt and Rampini (2006) have documented that the capital reallocation is procyclical while the cross-sectional dispersion of the productivity is countercyclical. Concluding from this funding, they suggest that the reallocation friction is countercyclical and we need a better foundation for that. Contrary to most macro models assuming exogenous adjustment costs, one advantage of our framework is allowing for a richer analysis of how this market friction responds to varied economic shocks and a better understanding of its aggregate implication.

First of all, we consider the shock to the underlying dispersion. From the previous analysis, it is clear that an increase in dispersion would increase the market frictions. Hence, the resulting resource mismatch would generate a drop in TFP and further increase the cross-sectional dispersion of productivity. It therefore provides an explanation for the coexistence of the countercyclical dispersion and procyclical reallocation, as documented in the empirical literature. It is definitely an interesting extension to endogenousize the underlying dispersion and worth further exploration.

In line with the growing literature on uncertainty, the shock to the downward uncertainty is also analyzed. Intuitively, a higher $\delta$, that is a higher level of instability of business condition would decrease investors' willingness to enter. Hence, the market liquidity also decreases with $\delta$, resulting in worse aggregate performance and a higher level of dispersion across firms. The story here, however, is different from Bloom (2009). 
Bloom (2009) shows that, with existence of capital adjustment costs, higher uncertainty (measured as a shock to the second moment) expands firms' inactive regions because it increases the real-option value of waiting. This concern then slows down the reallocations from low to high productivity firms. Instead of relying on an exogenous adjustment cost, firms who receive a negative shock, do want to exit but have a hard time finding an investor who is willing to buy their capital in our model. This idea also explains why few firms exit in bad time, as documented in Lee and Mukoyama (2008)

It is important to note that, however, an increase in the downward uncertainty, also results in a higher demand for the reallocation. Hence, the net effect on capital reallocation is ambiguous. As emphasized by Bachmann and Bayer (2009), a large countercyclical second moment shock would be incompatible with procyclical investment dispersion. Though, the shock on the downward uncertainty considered here is different from the second moment shock, it is important to understand that it also has two driving forces ${ }^{16}$ and that its net effect depends on assuming parameters. As a full calibration is beyond the scope of this paper, the main purpose of this exercise is to understand how uncertainty affects market frictions, as it can generate significant fluctuations in an environment with adverse selection.

\section{Conclusion}

(To be added)

\section{Appendix}

\subsection{Omitted Proof}

\section{(A) Proof of Proposition 1:}

Proof. One can observe that $U(\theta, s)$ satisfies following properties: that 1) $U_{2}(\theta, s)$ exists; 2) has an integrable bound: $\sup _{s \in S}\left|U_{2}(\theta, s)\right| \leq \frac{M}{r}$ for all s, where $M=u^{\prime}\left(s_{L}\right)$ for; 3) $U(\theta, \cdot)$ is absolutely continuos (as a function of $\mathrm{s}$ ) for all $\theta ; 4) \theta^{*}(s)$ is nonempty. Following the

\footnotetext{
${ }^{16}$ To see this, the total amount of reallocation at the steady state can be expressed as:$$
\frac{\delta}{\delta+m\left(\theta^{*}(s ; \delta)\right)} \cdot m\left(\theta^{*}(s ; \delta)\right)
$$ 
mechanism literature, (see Milgrom and Shannon (1994)), let

$$
V(s)=\max _{\hat{s}} U(\theta(\hat{s}), s)=\max \frac{u(s)+p(\theta(\hat{s})) m(\theta(\hat{s}))}{r+m(\theta(\hat{s}))}
$$

then any selection $\theta(s)$ from $\theta^{*}(s) \in \arg \max _{\theta^{\prime}} U\left(\theta^{\prime}, s\right)$,

$$
V(s)=V\left(s_{l}\right)+\int_{s_{l}}^{s} U_{s}\left(\theta^{*}(\tilde{s}), \tilde{s}\right) d \tilde{s}
$$

Namely, (ICFOC) is the necessary condition for any IC contract. To prove the sufficiency, define function: $x=q(\theta)=\frac{1}{r+m(\theta)}$ and $q^{-1}(x)=\theta$. Also, since $\theta>0$, $0<x \leqslant \frac{1}{r}$. One can then easily see $U(x, s)$ satisfies the strict single crossing difference property under the assumption $u^{\prime}(s)>0$. For any $x^{\prime}>x$ and $s^{\prime}>s$ :

$$
U\left(x^{\prime}, s^{\prime}\right)-U\left(x^{\prime}, s\right)+U(x, s)-U\left(x, s^{\prime}\right)=x^{\prime}\left(u\left(s^{\prime}\right)-u(s)\right)-x\left(u\left(s^{\prime}\right)-u(s)\right)>0
$$

Therefore, $U\left(x^{\prime}, s^{\prime}\right)-U\left(x, s^{\prime}\right)>U\left(x^{\prime}, s\right)-U(x, s)$. Given that $U(x, s)$ satisfies SSCD condition, then any non-decreasing $x(s)$ combined with $(I C F O C)$ are also sufficient conditions for the achievable outcome. Hence, $x(s)=\frac{1}{r+m\left(\theta^{*}(s)\right)}$ has to solve subject to the non-decreasing constraint. Namely, the market tightness function $\theta^{*}(\cdot)$ has to be nonincreasing.

\section{B) Proof of Lemma 2: No pooling}

Proof. Suppose Not: There exists a subset of sellers $s \in S^{\prime}=\left[s_{1}, s_{2}\right] \subset S$ are in the same market $\left(p_{\alpha}, \theta_{\alpha}\right)$.From the free entry condition,

$$
p_{\alpha}=\frac{E\left[s \mid s \in S^{\prime}\right]}{r}-\frac{k \theta_{\alpha}}{m\left(\theta_{a}\right)}
$$

and denote $V^{\alpha}\left(s_{2}\right)=V\left(p_{\alpha}, \theta_{\alpha}, s_{2}\right)$ as the utilities of the highest type seller in the market, and define the pair $\left(p_{2}, \theta_{2}\right)$ solves:

$$
\left\{\begin{array}{c}
p_{2}=\frac{s_{2}}{r}-\frac{k\left(\theta_{2}\right)}{m\left(\theta_{2}\right)} \\
V\left(p_{2}, \theta_{2}, s_{2}\right)=V^{\alpha}\left(s_{2}\right)
\end{array}\right\}
$$

Given $\left(p_{2}, \theta_{2}\right)$ solves above relations and $\frac{E\left[s \mid s \in S^{\prime}\right]}{r}<\frac{s_{2}}{r}$, there exists $p^{\prime}=p_{2}+\varepsilon$ such that $p_{\alpha}<p^{\prime}<p_{2}$ and $\theta^{\prime}$ which solve $V\left(p^{\prime}, \theta^{\prime}, s_{2}\right)=V^{\alpha}\left(s_{2}\right)$. From lemma $1, T\left(p^{\prime}\right)=s_{2}$. Namely, if a buyer deviates to posting $p^{\prime}$, only the highest type in the original pooling market will come. And, as explained, the expected market tightness is defined from (3), that is, $\theta^{\prime}=\theta\left(p^{\prime}\right)$.Obviously, $U_{b}\left(p^{\prime}, \theta^{\prime}, s_{2}\right)>U_{b}\left(p_{2}, \theta_{2}, s_{2}\right)=0$. Contradiction.

C) Proof of Lemma 3: the lowest type always receives his first best utility. 
Proof. Suppose not, pick any initial condition $\theta_{0}^{\prime} \in\left(0, \theta^{F B}\left(s_{L}\right)\right)$ and denote its corresponding market tightness as $\theta^{\prime}\left(s ; \theta_{0}^{\prime}\right)$ and price schedule $p^{\prime}(s)$. One can easily show that there exists $\tilde{p}=p^{\prime}\left(s_{L}\right)-\varepsilon$ and, from Lemma $1, T(\tilde{p})=s_{L}$. That is, a buyer can open a new market with lower price and expect the lowest type to come. Due to the violation of the tangent condition at $\left(p^{\prime}\left(s_{L}\right), \theta^{\prime}\left(s_{L}\right)\right)$ when $\theta^{\prime}\left(s_{L}\right) \neq \theta^{F B}\left(s_{L}\right)$ and $V^{\prime}\left(s_{L}\right)<V^{F B}\left(s_{L}\right)$, buyers' utility can be improved, $U\left(\tilde{p}, \theta(\tilde{p}), s_{L}\right)>\phi^{L}$. Contradiction.

\section{D) Proof of Heterogenous buyers}

Before proving the constructed solution is indeed the solution, we first prove that the following claim holds:

Claim $4 \forall \theta^{\prime}<\theta$, if $V\left(s^{\prime}, p, \theta\right)-V\left(s^{\prime}, p^{\prime}, \theta^{\prime}\right) \geq 0$ then $V(s, p, \theta)-V\left(s, p^{\prime}, \theta^{\prime}\right)>0$ for $\forall s^{\prime}>s$

Proof. $V(s, p, \theta)-V\left(s, p^{\prime}, \theta^{\prime}\right)=\left\{V\left(s^{\prime}, p, \theta\right)+\frac{u(s)-u\left(s^{\prime}\right)}{r+m(\theta)}\right\}-\left\{V\left(s^{\prime}, p^{\prime}, \theta^{\prime}\right)+\frac{u(s)-u\left(s^{\prime}\right)}{r+m\left(\theta^{\prime}\right)}\right\} \geq$ $\left(u\left(s^{\prime}\right)-u(s)\right)\left(\frac{1}{r+m\left(\theta^{\prime}\right)}-\frac{1}{r+m(\theta)}\right)>0$

Proof. a) Sellers' optimality: NTS: Given $(p(s), \theta(s)), s=\arg \max _{\hat{s}} V(s, p(\hat{s}), \theta(\hat{s}))$. First of all, we need to show that monotonic condition holds. The solution $\theta(s)$ is essentially the combination of $\theta(s ; l)$ and $\theta(s ; h)$, which are both non-increasing as long as the initial condition $\theta_{j}^{0}$ is smaller than $\theta_{j}^{F B}\left(s_{0}\right)$. Therefore, $\theta(s)$ is also non-increasing as long as $\theta_{H}^{0} \leq \theta\left(s^{*}, l\right)$, which holds by construction. From proposition 1 , it is clear that facing $(p(s, l), \theta(s, l))$, IC holds for sellers $s<s^{*}$, and, similarly, given $(p(s, h), \theta(s, h))$, IC holds for sellers $s \geq s^{*}$. What is left to prove is that those sellers will not benefit from entering the markets $\left\{p\left(s^{\prime}\right), \theta\left(s^{\prime}\right)\right\}$ for $\forall s^{\prime} \geq s^{*}$. Clearly, given that $V\left(s^{*}, p\left(s^{*}, l\right), \theta\left(s^{*}, l\right)=\right.$ $V\left(s^{*}, p\left(s^{*}, h\right), \theta\left(s^{*}, h\right)\right)>V\left(s^{*}, p\left(s^{\prime}, h\right), \theta\left(s^{\prime}, h\right)\right)$ and $\theta\left(s^{\prime}\right)<\theta\left(s^{*}\right)$ for all $s^{\prime} \geq s^{*}$, from claim 2:

$$
V(s, p(s), \theta(s))>V\left(s, p\left(s^{\prime}\right), \theta\left(s^{\prime}\right)\right) \text { for } \forall s^{\prime}>s
$$

Similarly, one can use the fact that $V\left(s^{*}, p\left(s^{*}, h\right), \theta\left(s^{*}, h\right) \geq V\left(s^{*}, p(s), \theta(s)\right)\right.$ and $\theta(s)<\theta\left(s^{\prime}\right)$ for all $s \leq s^{*}$ to prove $V\left(s^{\prime}, p\left(s^{\prime}\right), \theta\left(s^{\prime}\right)\right)>V\left(s^{\prime}, p(s), \theta(s)\right)$ for $\forall s<s^{*}$

b) Buyers' optimality: In order to make sure there is no profitable deviation for buyers, following two conditions much hold: b-1) $U_{b}\left(p(s), \theta(s), s, a^{j}\right)<\phi^{j}$ if $j^{*}(s) \neq$ $j$.Namely, given the markets which are already open, buyers will not enter the market to trade with seller $s$, if $j^{*}(s) \neq j$. Note that this is an additional condition we need to prove with heterogenous buyers; b-2) There does not exist $p^{\prime} \notin$ support $P$, such that $U_{b}\left(p^{\prime}, \theta\left(p^{\prime}\right), s^{\prime}, a^{j}\right)<\phi^{j}$, where $\theta\left(p^{\prime}\right)$ is defined as (3) and $s^{\prime} \in T_{s}$ is the type of a seller who 
is most likely to come. That is, the buyer will not benefit from posting a price $p^{\prime}$ which is not recommended by the market maker.

b-1) First of all, we show that low type of buyers will not enter the market with sellers $s$, where $j^{*}(s)=h$. Observe that by construction, $\theta\left(s ; h, \theta_{H}^{0}\right)<\tilde{\theta}(s)$ for $\forall s>s^{*}$. This is true as long as $\theta_{H}^{0} \leq \tilde{\theta}\left(s^{*}\right)$, given that $\theta\left(s ; h, \theta_{H}^{0}\right)$ decreases with s and $\tilde{\theta}(s)$ increases with s. The condition is satisfied since, by construction, if $\tilde{\theta}\left(s^{A}\right) \geq \theta_{H}^{F B}\left(s^{A}\right), \theta_{H}^{0}=\theta_{H}^{F B}\left(s^{A}\right) \leq \tilde{\theta}\left(s^{A}\right)$; otherwise, $\theta_{H}^{0}=\tilde{\theta}\left(s^{B}\right)$. Hence, from claim 1, low type will not enter the market with the pair $\left\{p^{*}(s), \theta^{*}(s)\right\}$ for $\forall s>s^{*}$. Similarly, by construction, $\theta\left(s ; l, \theta_{L}^{0}\right) \geq \theta(s)$ for $\forall s \leq s^{*}$. Hence, high-type buyers will not enter the the market with the pair $\left\{p^{*}(s), \theta^{*}(s)\right\}$ for $\forall s<s^{*}$

b-2) Let $\{\bar{p}, \underline{p}$ \}as the upper bound and the lower bound of the support constructed price schedule $P$. Apply Lemma 1 , for any new posting $p^{\prime}>\bar{p}, T_{s}\left(p^{\prime}\right)=s_{H}$ and $p^{\prime}<\underline{\mathrm{p}}$, $T_{s}\left(p^{\prime}\right)=s_{L}$. Obviously, the lower type will not benefit from opening $p^{\prime}<\underline{\mathrm{p}}$ since $\left(p^{*}, \theta^{*}\right)$ is the first-best solution. The higher type obviously will not benefit from attracting the lowest type seller, given $V^{F B}(s, h)<V^{F B}(s, l)$ and, therefore, $U_{b}\left(p^{\prime}, \theta^{\prime}, s_{L} ; V^{F B}\left(s_{L}, l\right)\right)<$ $\phi^{h}$. Similarly, as before, neither the high type will benefit from posting $p^{\prime}>\bar{p}$ to attract the highest type with a even higher distortion of market liquidity, nor the lower type buyer. Notice that $p(\cdot)$ has a jump discontinuity at $s^{*}$ when $s^{*}=s^{A}$. In particular, there are two markets opened for the seller $s^{*}$, and, among two of them, sellers are indifferent. Given any $p^{\prime} \in\left(p\left(s^{*}, l\right), p\left(s^{*}, h\right)\right), T\left(p^{\prime}\right)=s^{*}$ according to Lemma 1. Given that the type who is most likely come is $s^{*}$, it is clear that low type buyer will not raise the price $p^{\prime}>p\left(s^{*}, l\right)$ to attract the same seller. Nor the high type will benefit from posting $p^{\prime}<$ $p\left(s^{*}, h\right)$ since the pair of $p\left(s^{*}, h\right)$ and $\theta\left(s^{*}, l\right)=\theta_{H}^{F B}(s)$ has already maximized the joint surplus (first best).

Proof. Uniqueness: From Lemma 3, we know that $\theta_{L}^{0}=\theta_{L}^{F B}(s)$. Hence, to prove the uniqueness, we essentially need to show that marginal types $s^{*}$ constructed above and its corresponding $\theta_{H}^{0}$ is unique. First of all, by construction, $V_{H}^{F B}(s)<V(s, l)$ for $\forall s<s^{A}$, it is clear that a high-type buyer will not enter the market for $s<s^{A}$, given that the highest utilities he can offer to the seller is lower than the one offered by a low type buyer. Also, from Claim 1, $s^{*}$ can not be larger that $s^{B}$ in the equilibrium otherwise there exists $s<s^{*}$ such that $\theta(s, l)<\tilde{\theta}(s)$, which implies that it is profitable for a higher type to enter this market. Hence, the only possible range is $\left[s^{A}, s^{B}\right]$. Given that there are two different cases, depending on the relation between $\tilde{\theta}\left(s_{A}\right)$ and $\theta_{H}^{F B}\left(s_{A}\right)$, we will prove the uniqueness separately for each case. Before that, we first prove formally that $s^{A}$ is unique. Notice that $s^{A}$ is the intersection of $V_{H}^{F B}(s)$ and $V(s, l)$. Therefore, the unique of $s^{A}$ is 
obtained as long as $V_{H}^{F B}(s)-V(s, l)$ satisfies single crossing condition. As a result of the following inequalities, we can conclude that $V_{H}^{F B}\left(h, s^{\prime}\right)-V\left(l, s^{\prime}\right)>V_{H}^{F B}(h, s)-V(l, s)$ for any $s^{\prime}>s$

$$
\begin{aligned}
& V_{H}^{F B}\left(s^{\prime}\right)-\left(V_{L}^{F B}(s)-V(l, s)\right)-V\left(l, s^{\prime}\right) \\
> & V_{H}^{F B}\left(s^{\prime}\right)-\left(V_{L}^{F B}\left(s^{\prime}\right)-V\left(l, s^{\prime}\right)\right)-V\left(l, s^{\prime}\right) \\
> & V_{H}^{F B}(s)-\left(V_{L}^{F B}(s)-V(l, s)\right)-V(l, s)
\end{aligned}
$$

The first inequality follows from the fact that $V_{L}^{F B}\left(s^{\prime}\right)-V\left(l, s^{\prime}\right)>V_{L}^{F B}(s)-V(l, s)$, that is, the utility of a high type seller decreases more than the one of a low type, resulting from a higher distortion ${ }^{17}$. The second inequality follows from the condition of PAM, that's, $V_{H}^{F B}\left(s^{\prime}\right)-V_{L}^{F B}\left(s^{\prime}\right)>V_{H}^{F B}(s)-V_{L}^{F B}(s)$. Moreover, from the discussion of b-2), we can conclude the following claim: (Claim 3) If there is a discontinuity in $\theta^{*}(\cdot)$ at $s^{*}$, which necessarily induces a discontinuity in $p(\cdot)$, it has to be the case that $\theta_{H}^{0}=\theta_{H}^{F B}\left(s^{*}\right)$. Namely, $\theta_{H}^{0}$ must equal its first best value $\theta_{H}^{F B}\left(s^{*}\right)$ when $\theta_{H}^{0} \neq \theta\left(s^{*}, l\right)$; otherwise, there is a profitable deviation for a high type buyer, who will deviate by posting a new price $p^{\prime}=p\left(\theta_{H}^{0}\right)-\varepsilon \notin$ range of $P$ to attract $s^{*}$.

$(\mathrm{CASE} 1) s^{*}=s^{A}$ when $\tilde{\theta}\left(s^{A}\right) \geq \theta_{H}^{F B}\left(s^{A}\right): \tilde{\theta}\left(s^{A}\right) \geq \theta_{H}^{F B}\left(s^{A}\right)$ immediately implies that $\theta\left(s^{A}, l\right) \geq \tilde{\theta}\left(s^{A}\right) \geq \theta_{H}^{F B}\left(s^{A}\right)^{18}$ and the equality holds iff $\tilde{\theta}\left(s^{A}\right)=\theta_{H}^{F B}\left(s^{A}\right)$. Pick $s_{m} \in$ $\left(s^{A}, s^{B}\right]$ as the marginal type. By definition, the marginal type must be indifferent among two markets, that is, indifferent between $\left(\theta\left(s_{m}, l\right), p\left(s_{m}, l\right)\right)$ and $\left(\theta_{H}^{0}, p\left(s_{m}, h\right)\right)$. Given that $s_{m}>s^{A} \Longrightarrow V_{H}^{F B}\left(s_{m}\right)>V\left(s_{m}, l\right)$, It has to be case that $\theta_{H}^{0}<\theta_{H}^{F H}\left(s_{m}\right)$, that is, there must be a downward distortion ${ }^{19}$ in market tightness otherwise $s_{m}$ is strictly better off going to the market with a high type buyer. According to Claim 3, this can not be sustained in equilibrium if there is a discontinuous in $\theta^{*}(\cdot)$. Namely, the only possible case is that $\theta_{H}^{0}=\theta\left(s_{m}, l\right)<\theta_{H}^{F B}\left(s^{m}\right)$. However, given that $\theta\left(s^{A}, l\right) \geq \theta_{H}^{F B}\left(s^{A}\right), \theta\left(s_{m}, l\right)$ decreases with $s$ and $\theta_{H}^{F B}(s)$ increases with $\mathrm{s}, \theta\left(s_{m}, l\right)<\theta_{H}^{F B}\left(s^{m}\right) \Longrightarrow \exists s^{\prime} \in\left(s^{A}, s^{m}\right)$ such that $\theta\left(s^{\prime}, l\right)=\theta_{H}^{F B}\left(s^{\prime}\right)>\tilde{\theta}\left(s^{\prime}\right)$. The above relation implies that $V\left(p^{l}\left(s^{\prime}\right), \theta\left(s^{\prime}, l\right)\right)>V_{H}^{F B}\left(s^{\prime}\right)$,

${ }^{17}$ One can show that $\theta_{L}^{F B}(s)-\theta^{*}(s, l)$ increases with $s$, given that $\theta_{L}^{F B}(s)$ increases with s while $\theta^{*}(s, l)$ decreases with s.

${ }^{18}$ Note that $\theta\left(s^{A}, l\right)$ is the intersection of a low-type buyer's utility at $\phi^{l}$ and the utility of a seller $s^{A}$ with the level of $V_{H}^{F B}\left(s^{A}\right)$. Given that $\tilde{\theta}\left(s^{A}\right) \geq \theta_{H}^{F B}\left(s^{A}\right)$ and the tangent condition of $V_{H}^{F B}\left(s^{A}\right)$ and the utility of a high-type buyer is satisfied, $\theta\left(s^{A}, l\right)$ must be larger than $\tilde{\theta}\left(s^{A}\right)$. This is because that, by construction, the utility curve of $U_{b}(s, p, \theta)=\phi^{l}$ lies below $U_{b}(s, p, \theta)=\phi^{h}$ for any $\theta<\tilde{\theta}(s)$ and above $U_{b}(s, p, \theta)=\phi^{h}$ for any $\theta>\tilde{\theta}(s)$.

${ }^{19}$ Clearly, it has to be downward distortion instead of upward one since the monotonic condition from sellers' IC won't hold for any $\theta_{H}^{0}>\theta_{H}^{F B}(s)$. 
which contradicts with the fact that $V_{H}^{F B}\left(h, s^{\prime}\right)-V\left(l, s^{\prime}\right)>0$ for $\forall s^{\prime}>s^{A}$. Therefore, we show that $s^{*}=s^{A}$ is the unique solution when $\tilde{\theta}\left(s^{A}\right) \geq \theta_{H}^{F B}\left(s^{A}\right)$.

$\left(\right.$ CASE 2) $s^{*}=s^{B}$ when $\tilde{\theta}\left(s^{A}\right)<\theta_{H}^{F B}\left(s^{A}\right)$. First of all, $\tilde{\theta}\left(s^{A}\right)<\theta_{H}^{F B}\left(s^{A}\right)$ implies that $\theta_{H}^{F B}\left(s^{A}\right)>\theta\left(s^{A}, l\right)>\tilde{\theta}\left(s^{A}\right)$. Same as before, the only possible range for $s_{m}$ is $\left[s^{A}, s^{B}\right)$. Given that $\tilde{\theta}(s)$ and $\theta_{H}^{F B}(s)$ increase with s, while $\theta(s, l)$ decreases with s and $\theta_{H}^{F B}\left(s^{A}\right)>$ $\theta\left(s^{A}, l\right)>\tilde{\theta}\left(s^{A}\right)$, it has to be the case that $\theta_{H}^{F B}\left(s_{m}\right)>\theta\left(s_{m}, l\right)>\tilde{\theta}\left(s_{m}\right)$ for $\forall s_{m} \in\left[s^{A}, s^{B}\right)$. Moreover, according to Claim 1 , the initial condition $\theta_{H}^{0}$ has to smaller $\tilde{\theta}\left(s_{m}\right)$. Therefore, $\theta\left(s_{m}, l\right)>\tilde{\theta}\left(s_{m}\right) \geq \theta_{H}^{0}$, which necessarily results in a discontinuity of $\theta^{*}(\cdot)$ at $s_{m}$. The resulting discontinuity and $\theta_{H}^{0}<\theta_{H}^{F B}\left(s_{m}\right)$ violates Claim 3. Contradictions. The above argument also confirms why only $s^{B}$ and $\theta_{H}^{0}=\theta\left(s^{B}, l\right)=\tilde{\theta}\left(s^{B}\right)$ is the unique solution in this case, guaranteeing the continuity of $\theta^{*}(s)$.

E) Proof of Claim 1 and Claim 2: From the FOC of the first best solution, one can solve $\frac{d \theta^{F B}(s)}{d s}=\frac{h^{\prime}(s)-u^{\prime}(s)}{k\left(\frac{1-\rho}{\rho}\right)\left(1+\theta^{-\rho}\right)} \equiv f_{2}(\theta, s)$. Observe from the differential equation, $\frac{d \theta^{*}(s)}{d s}=f(\theta, s) \rightarrow-\propto$ at $f\left(\theta^{F B}(s), s\right)$ given $h_{s}>0$. Hence, we know that $\theta^{*}(s) \leq \theta^{F B}(s)$ for some $s_{1}>s_{L}$. Suppose now $\theta^{*}(s)>\theta^{F B}(s)$ for some $s$, which implies that these two function must cross at some point $\left(\hat{s}, \theta^{F B}(\hat{s})\right)$ and the slope of the crossing point must be the case that $f_{2}\left(\theta^{F B}(\hat{s}), \hat{s}\right)<f\left(\theta^{F B}(\hat{s}), \hat{s}\right)=-\propto$. Contradiction.

\section{F) Proof of Proposition 6 (to be added)}

\section{References}

Akerlof, G. (1970). The market for lemons. Quarterly journal of Economics $84(3)$, $488-500$.

Bachmann, R. and C. Bayer (2009). The cross-section of firms over the business cycle: new facts and a DSGE exploration. Dt. Bundesbank, Press and Public Relations Div.

Bloom, N. (2009). The impact of uncertainty shocks. Econometrica 7ry(3), 623-685.

Caballero, R. (2007). Specificity and the Macroeconomics of Restructuring. The MIT Press.

Chiu, J. and T. Koeppl (2010). Market freeze and recovery: Trading dynamics under optimal intervention by a market-maker-of-last-resort. Technical report, Citeseer.

Duffie, D., N. Garleanu, and L. Pedersen (2005). Over-the-counter markets. Econometrica $73(6)$, 1815-1847. 
Duffie, D., N. Gârleanu, and L. Pedersen (2007). Valuation in over-the-counter markets. Review of Financial Studies 20(6), 1865.

Eeckhout, J. and P. Kircher (2010). Sorting and decentralized price competition. Econometrica 78(2), 539-574.

Eisfeldt, A. and A. Rampini (2006). Capital reallocation and liquidity. Journal of Monetary Economics 53(3), 369-399.

Gale, D. (1992). A Walrasian theory of markets with adverse selection. The Review of Economic Studies, 229-255.

Gale, D. (1996). Equilibria and Pareto optima of markets with adverse selection. Economic Theory 7(2), 207-235.

Gavazza, A. (2010). The role of trading frictions in real asset markets. American Economic Review.

Guerrieri, V., R. Shimer, and R. Wright (2009). Adverse selection in competitive search equilibrium. NBER Working Paper.

Guerrieri, V., R. Shimer, and R. Wright (2010). Competitive Equilibrium in Asset Markets with Adverse Selection.

Lagos, R. and G. Rocheteau (2009). Liquidity in asset markets with search frictions. Econometrica $77(2), 403-426$.

Lee, Y. and T. Mukoyama (2008). Entry, exit and plant-level dynamics over the business cycle.

Maksimovic, V. and G. Phillips (2001). The market for corporate assets: Who engages in mergers and asset sales and are there efficiency gains? The Journal of Finance 56(6), 2019-2065.

Milgrom, P. and C. Shannon (1994). Monotone comparative statics. Econometrica: Journal of the Econometric Society 62(1), 157-180.

Moen, E. (1997). Competitive search equilibrium. Journal of Political Economy 105(2), 385-411.

Mortensen, D. and R. Wright (2002). Competitive Pricing and Efficiency in Search Equilibrium*. International Economic Review 43(1), 1-20.

Shi, S. (2001). Frictional Assignment. I. Efficiency* 1. Journal of Economic Theory 98(2), 232-260. 
Tirole, J. (2010). Illiquidity and all its friends. Fondazione Eni Enrico Mattei Working Papers, 467.

Tirole, J. (2011). Overcoming Adverse Selection: How Public Intervention Can Restore Market Functioning.

Warusawitharana, M. (2008). Corporate asset purchases and sales: Theory and evidence. Journal of Financial Economics 87(2), 471-497.

Weill, P. (2008). Liquidity premia in dynamic bargaining markets. Journal of Economic Theory 140(1), 66-96. 